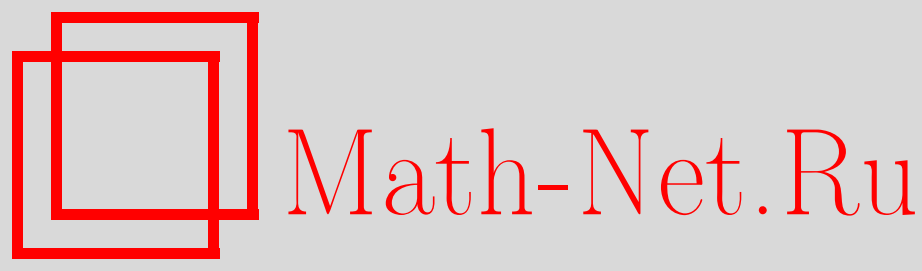

Д. С. Широков, Теорема Паули при описании $n$-мерных спиноров в формализме алгебр Клиффорда, ТМФ, 2013, том 175, номер 1, 11-34

DOI: https://doi.org/10.4213/tmf8384

Использование Общероссийского математического портала Math-Net.Ru подразумевает, что вы прочитали и согласны с пользовательским соглашением http://www . mathnet.ru/rus/agreement

Параметры загрузки:

IP : 54.237 .59 .107

26 апреля 2023 г., 16:51:44

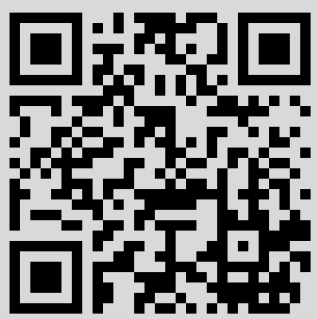




\title{
ТЕОРЕМА ПАУЛИ ПРИ ОПИСАНИИ $n$-МЕРНЫХ СПИНОРОВ В ФОРМАЛИЗМЕ АЛГЕБР КЛИФФОРДА
}

\begin{abstract}
Обсуждается обобщенная теорема Паули и ее возможные применения при описании $n$-мерных спиноров (Дирака, Вейля, Майорана и Майорана-Вейля) в формализме алгебр Клиффорда. Предложен явный вид элементов, осуществляющих обобщения дираковского, зарядового и майорановского сопряжений в случае произвольных размерностей и сигнатур пространства. При описании сопряжений используется понятие дополнительной сигнатуры алгебры Клиффорда. Показано, что дополнительная сигнатура может принимать только определенные значения, несмотря на зависимость от матричного представления.
\end{abstract}

Ключевые слова: теорема Паули, спиноры, алгебра Клиффорда, дираковское сопряжение, зарядовое сопряжение, майорановское сопряжение, спиноры Вейля, спиноры Майорана, спиноры Майорана-Вейля, дополнительная сигнатура алгебры Клиффорда.

DOI: $10.4213 / \operatorname{tmf} 8384$

\section{1. ВВЕДЕНИЕ}

В 1936 году Паули [1] доказал так называемую фундаментальную теорему о гамма-матрицах Дирака. Теорема утверждает, что два набора из четырех квадратных комплексных матриц четвертого порядка, которые антикоммутируют между собой и квадраты которых равны единичной матрице или единичной матрице с обратным знаком, связаны преобразованием подобия, причем матрица подобия единственна с точностью до умножения на ненулевое комплексное число. Эта теорема играет важную роль при изучении различных вопросов, возникающих в теории поля (см., например, [2], [3]). В частности, с помощью теоремы Паули доказывается лоренц-инвариантность уравнения Дирака, описывается связь спинорных и ортогональных групп, вводится понятие спиноров Майорана.

Имеются общеизвестные утверждения, которые в некотором смысле обобщают теорему Паули на случай произвольной размерности. А именно, методами теории представлений несложно показать [4], [5], что алгебра Клиффорда имеет единственное (с точностью до эквивалентности) неприводимое представление в случае четной

* Математический институт им. В. А. Стеклова РАН, Москва, Россия. E-mail: shirokov@mi.ras.ru 
размерности и два неприводимых представления в случае нечетной размерности. Данные утверждения применяются в различных задачах математической физики, в частности в теории суперсимметрии (см. [5]-[19]).

В одной из предыдущих работ [20] автором были предложены утверждения, обобщающие теорему Паули. А именно, рассматривается более общий вопрос (который не всегда сводится к рассмотрению представлений) о связи двух наборов элементов алгебр Клиффорда, удовлетворяющих определяющим антикоммутационным соотношениям. Даются обобщения на случай алгебр Клиффорда произвольных четных и нечетных размерностей над полем вещественных и комплексных чисел. Показано, что в нечетном вещественном случае есть четыре (а в комплексном шесть) варианта связи между двумя наборами элементов, удовлетворяющих антикоммутационным соотношениям алгебры Клиффорда. В отличие от теоремы Паули в случае четырехмерного пространства Минковского, где связь осуществляется преобразованием подобия, в случае произвольной нечетной размерности (например, в случае трехмерного пространства) два набора связаны преобразованием подобия с точностью до умножения на элемент алгебры Клиффорда $\beta^{1 \ldots n} \gamma_{1 \ldots n}$, который может принимать в вещественном случае четыре (в комплексном случае шесть) различных значения (см. ниже теоремы 5,6 ). Кроме того, во всех случаях (четной и нечетной размерностей) указаны явные алгоритмы вычисления элемента, осуществляющего эту связь.

В настоящей работе мы также указываем возможные приложения доказанных теорем в различных задачах математической физики. Стоит отметить несколько направлений, связанных с применением обобщенной теоремы Паули (OTП).

Первым направлением можно назвать изучение $n$-мерного уравнения Дирака, в частности вопрос об инвариантности уравнения при псевдоортогональных (в частном случае лоренцевых) преобразованиях координат. В настоящее время активно используется трехмерное уравнение Дирака для графена. Таким образом, уравнение Дирака представляет интерес не только в случае четных, но и в случае нечетных размерностей. Локальная ОТП используется при изучении систем уравнений Дирака-Максвелла и Дирака-Янга-Миллса [21].

Второе применение относится к изучению связи спинорных и ортогональных групп. Мы предлагаем альтернативное доказательство с помощью ОТП теоремы о двулистных накрытиях ортогональных групп спинорными в случае произвольных размерностей (без использования теоремы Картана-Дьедонне, как делается в стандартном изложении). Кроме того, мы предлагаем явные алгоритмы вычисления элементов спинорных групп, которым соответствуют элементы ортогональных групп при двулистном накрытии.

Третье возможное применение, на котором мы и остановимся в настоящей работе, возникает при изучении $n$-мерных спиноров. Мы даем описание элементов, осуществляющих обобщения дираковского, майорановского и зарядового сопряжений от спинора в случае произвольных размерностей и сигнатур пространства. Отметим, что в случае четных размерностей рассмотрено по два аналога сопряжения каждого вида. В связи с вопросом о существовании спиноров Дирака, Вейля, Майорана и Майорана-Вейля в формализме алгебр Клиффорда в случае различных размерностей и сигнатур пространства возникают вопросы, связанные с применением 
ОТП в теории суперсимметрии (отметим классические работы по суперсимметрии и супергравитации Шерка, Глиоззи, Оливе [9], Куго и Таундсена [8], современные обзоры [5], [6] и другие работы [10]-[19]). В настоящей работе при изучении подобных вопросов мы используем ОТП.

Отметим, что мы используем аппарат алгебр Клиффорда над полем вещественных и комплексных чисел произвольных размерностей и сигнатур. Этот аппарат представляется нам более естественным и удобным (например, по сравнению с матричным аппаратом) при изучении перечисленных выше вопросов. При описании спиноров существенную роль играет структура алгебр Клиффорда, которая подчиняется 8-периодичности Картана-Ботта (см. ниже теорему 2).

В разделе 2 дается определение алгебры Клиффорда, рассмотрены связанные с ней понятия, которые необходимы в дальнейшем изложении. Кроме того, сформулированы общеизвестные теоремы о центре алгебры Клиффорда и об изоморфизмах алгебр Клиффорда матричным алгебрам. Далее, в разделе 3 сформулированы утверждения, обобщающие теорему Паули на случай вещественных и комплексных алгебр Клиффорда произвольных размерностей. Эти теоремы играют ключевую роль в дальнейшем изложении.

В разделе 4 дается краткий обзор $n$-мерных спиноров Дирака и Вейля в формализме алгебр Клиффорда.

В разделах 5, 6 обсуждается вопрос о согласованности операций над элементами алгебры Клиффорда и операций над матрицами. При изучении этого вопроса активно используется ОТП. Представлены новые результаты (теоремы 7, 8). Теорема 7 является следствием ОТП (теоремы 4, 5, 6). В теореме 8 предлагаются явные формулы для связи матричных операций с операциями в алгебре Клиффорда (при этом используется введенное понятие дополнительной сигнатуры алгебры Клиффорда, которая зависит от матричного представления). На основе этих формул ниже предлагаются явные формулы для элементов, осуществляющих обобщения дираковского, майорановского и зарядового сопряжений.

В разделах 7-9 доказываются теоремы об аналогах дираковского, майорановского и зарядового сопряжений соответственно в случае произвольных размерностей и сигнатур пространства. Здесь же приводятся общеизвестные утверждения о реализации $n$-мерных спиноров Майорана, псевдомайорановских спиноров и спиноров Майорана-Вейля (выкладки проводятся в формализме алгебр Клиффорда). Доказывается теорема о том, что дополнительная сигнатура алгебры Клиффорда может принимать ограниченное число значений, несмотря на зависимость от матричного представления (теорема 12).

Отметим, что некоторые аспекты, связанные с $n$-мерными спинорами, представлены (иногда в другом формализме) в литературе. Мы постарались дать более полное математическое описание теории $n$-мерных спиноров Вейля, Майорана и Майорана-Вейля в случае произвольных размерностей и сигнатур. Излагается своя точка зрения на поставленные вопросы, представлено несколько новых результатов (теоремы 7-9, 11, 12, 14). В теоремах 9, 11, 14 даны явные формулы для элементов алгебры Клиффорда $A_{ \pm}, B_{ \pm}$и $C_{ \pm}$, которые явно зависят от матричного представления алгебры Клиффорда. При этом используется понятие дополнительных сигнатур алгебр Клиффорда $\left(k_{\gamma}, l_{\gamma}\right)$ и $\left(r_{\gamma}, s_{\gamma}\right)$. 
Ключевую роль в наших рассмотрениях играют ОТП (теоремы 4-6), с помощью которых мы связываем операции над матрицами с операциями над элементами алгебры Клиффорда.

\section{2. МАТЕМАТИЧЕСКИЙ АППАРАТ АЛГЕБР КЛИФФОРДА}

Алгебра Клиффорда (первоначальное название - геометрическая алгебра) была открыта английским математиком Вильямом Клиффордом [22] в 1878 году как алгебра, объединяющая свойства алгебры Грассмана [23] и кватернионов Гамильтона [24].

Дальнейшее развитие алгебр Клиффорда связано с целым рядом известных математиков и физиков (Р. Липшиц, Т. Вален, Э. Картан, Э. Уитт, К. Шевалле [25], М. Рис [26] и др.). Существенным для изучения алгебр Клиффорда оказалось открытие в 1928 году уравнения Дирака [27] для электрона, к которому алгебра Клиффорда имеет непосредственное отношение. Уравнение Дирака записывается с использованием четырех комплекснозначных матриц (гамма-матриц Дирака), которые удовлетворяют тем же определяющим соотношениям, что и генераторы алгебры Клиффорда $\mathcal{C}(1,3)$. Связь алгебры Клиффорда со спинорами привлекла внимание к теории алгебр Клиффорда со стороны многих физиков и математиков.

В настоящее время алгебры Клиффорда применяются во многих разделах современной математики и физики. Например, алгебра Клиффорда находит свое применение в теории поля [28], [29], робототехнике, обработке сигналов и изображений, химии, небесной механике, вычислительной технике, электродинамике, геометрии и др.

В литературе известно несколько различных (эквивалентных) определений алгебр Клиффорда ${ }^{1)}$. В рассматриваемом далее определении алгебры Клиффорда используется базис специального вида - занумерованный упорядоченными мультииндексами. Подчеркнем, что введенные ниже генераторы и базис фиксированы (не меняются). Такое определение ближе к первоначальному определению Клиффорда.

Пусть $E$ - векторное (линейное) пространство над полем $\mathbb{F}$ вещественных чисел $\mathbb{R}$ или над полем комплексных чисел $\mathbb{C}$. Пусть $n$ - натуральное число, размерность пространства $E$ равна $\operatorname{dim} E=2^{n}$. Пусть в $E$ введен базис

$$
e, e^{a}, e^{a_{1} a_{2}}, \ldots, e^{1 \ldots n}, \quad a_{1}<a_{2}<\cdots \quad\left(2^{n} \text { штук }\right)
$$

занумерованный упорядоченными мультииндексами длины от 0 до $n$. Индексы $a, a_{1}, a_{2}, \ldots$ пробегают значения от 1 до $n$.

Пусть $p$ и $q$ - неотрицательные целые числа и $p+q=n, n \geqslant 1$. Введем диагональную матрицу $\eta$ размера $n: \eta=\left\|\eta^{a b}\right\|=\operatorname{diag}(1, \ldots, 1,-1, \ldots,-1)$, у которой на диагонали стоят $p$ штук +1 и $q$ штук -1 . Введем на $E$ операцию клифбордова

1) В монографии [30] рассмотрены сразу три различных определения алгебры Клиффорда и показана их эквивалентность. В разных случаях бывают удобны разные определения. После работ Картана определение алгебры Клиффорда дается как определение алгебры Клиффорда $n$-мерного векторного пространства $V$ с заданной квадратичной формой $Q$ сигнатуры $(p, q)$, где $n=p+q$. При этом требуется, чтобы алгебра Клиффорда содержала изометричную копию $V$. 
умножения $U, V \rightarrow U V$ так, что выполнены свойства дистрибутивности, ассоциативности, $e$ - единичный элемент,

$$
\begin{aligned}
e^{a} e^{b}+e^{b} e^{a} & =2 \eta^{a b} e & & \forall a, b=1, \ldots, n, \\
e^{a_{1}} \ldots e^{a_{k}} & =e^{a_{1} \ldots a_{k}}, & & 1 \leqslant a_{1}<\cdots<a_{k} \leqslant n .
\end{aligned}
$$

Тогда введенная таким образом алгебра называется алгеброй Клиффорда и обозначается $\mathcal{C} \ell^{\mathbb{R}}(p, q)$ в случае поля вещественных чисел и $\mathcal{C} \ell^{\mathbb{C}}(p, q) \equiv \mathcal{C}(p, q)$ в случае поля комплексных чисел ${ }^{2}$. Если рассуждения верны для обоих случаев, будем писать $\mathcal{C} \ell^{\mathbb{F}}(p, q)$, подразумевая, что $\mathbb{F}=\mathbb{R}$ или $\mathbb{F}=\mathbb{C}$.

Элементы $e^{a}$ называются генераторами ${ }^{3)}$ алгебры Клиффорда, элемент $е$ называется единицей алгебры Клиффорда. Пара чисел $(p, q)$ называется сигнатурой алгебры Клиффорда $\mathcal{C} \ell^{\mathbb{F}}(p, q)$. Заметим, что часто сигнатурой также называется число $p-q$.

Любой элемент $U$ алгебры Клиффорда $\mathcal{C} \mathbb{F}^{\mathbb{F}}(p, q)$ представляется в виде разложения по базису

$$
U=u e+u_{a} e^{a}+\sum_{a_{1}<a_{2}} u_{a_{1} a_{2}} e^{a_{1} a_{2}}+\cdots+u_{1 \ldots n} e^{1 \ldots n}
$$

где $u, u_{a}, u_{a_{1} a_{2}}, \ldots, u_{1 \ldots n}-$ вещественные (в случае $\left.\mathcal{C} \ell^{\mathbb{R}}(p, q)\right)$ или комплексные (в случае $\mathcal{C}(p, q))$ числа.

Векторные подпространства, натянутые на элементы $e^{a_{1} \ldots a_{k}}$, занумерованные упорядоченными мультииндексами длины $k$, обозначаются через $\mathcal{C} \ell_{k}^{\mathbb{F}}(p, q)$. Элементы подпространства $\mathcal{C} \ell_{k}^{\mathbb{F}}(p, q)$ называются элементами ранга $k$. Отметим, что

$$
\operatorname{dim} \mathcal{C} \ell_{k}^{\mathbb{F}}(p, q)=C_{n}^{k}
$$

Разбиение $\mathcal{C} \ell^{\mathbb{F}}(p, q)=\bigoplus_{k=0}^{n} \mathcal{C} \ell_{k}^{\mathbb{F}}(p, q)$ задает классификацию элементов алгебр Клиффорда по рангам.

$Z_{2}$-градуировка. Алгебра Клиффорда $\mathcal{C} \ell^{\mathbb{F}}(p, q)$ является $Z_{2}$-градуированной алгеброй (супералгеброй), а именно представляется в виде прямой суммы с соответствующими свойствами для четного и нечетного подпространств:

$$
\mathcal{C} \ell^{\mathbb{F}}(p, q)=\mathcal{C} \ell_{\text {even }}^{\mathbb{F}}(p, q) \oplus \mathcal{C} \ell_{\text {odd }}^{\mathbb{F}}(p, q)=\underset{k-\text { even }}{\bigoplus} \mathcal{C} \ell_{k}^{\mathbb{F}}(p, q) \oplus \underset{k-\text { odd }}{\bigoplus} \mathcal{C} \ell_{k}^{\mathbb{F}}(p, q)
$$

Рассмотрим следующие операции сопряжения от элементов алгебры Клиффорда, которые нам понадобятся в дальнейшем.

Комплексное сопряжение. Если элемент $U \in \mathcal{C} \ell(p, q)$ задан в виде разложения (1), то операцию комплексного сопряжения от элемента алгебры Клиффорда

2) Заметим, что комплексные алгебры Клиффорда одной размерности $n$ (и разных сигнатур) изоморфны друг другу как алгебры (см. теорему 3). Однако в приложениях рассматриваются комплексные алгебры Клиффорда различных сигнатур. Например, при рассмотрении уравнения Дирака используется комплексная алгебра Клиффорда $\mathcal{C} \mathbb{C}^{\mathbb{C}}(1,3)$ (а не $\left.\mathcal{C} \mathbb{C}^{\mathbb{C}}(4,0)\right)$. Комплексные алгебры Клиффорда $\mathcal{C} \mathbb{C}^{\mathbb{C}}(p, q)$ целесообразно рассматривать в тех задачах, в которых требуется использовать операцию комплексного сопряжения от элементов алгебры Клиффорда.

3) В русскоязычной литературе вместо термина "генератор" часто используется термин "порождающий". 
$U \rightarrow \bar{U}$ зададим формулой

$$
\bar{U}=\bar{u} e+\bar{u}_{a} e^{a}+\sum_{a_{1}<a_{2}} \bar{u}_{a_{1} a_{2}} e^{a_{1} a_{2}}+\sum_{a_{1}<a_{2}<a_{3}} \bar{u}_{a_{1} a_{2} a_{3}} e^{a_{1} a_{2} a_{3}}+\cdots,
$$

в которую входят комплексно-сопряженные коэффициенты $\bar{u}, \bar{u}_{a}, \bar{u}_{a_{1} a_{2}}, \ldots$

В соответствии с этой формулой элементы базиса алгебры Клиффорда рассматриваются как вещественные величины, т. е. $\bar{e}^{a_{1} \ldots a_{k}}=e^{a_{1} \ldots a_{k}}$. Равенства $\overline{\bar{U}}=U$, $(\overline{U V})=\bar{U} \bar{V},(\overline{U+V})=\bar{U}+\bar{V},(\overline{\lambda U})=\bar{\lambda} \bar{U}$ верны для произвольных элементов $U, V \in \mathcal{C} \ell(p, q)$ и чисел $\lambda \in \mathbb{C}$.

Реверс. Для элемента $U \in \mathcal{C} \ell(p, q)$ определим операцию сопряжения $U \rightarrow U^{\sim}$, называемую реверсом:

$$
U^{\sim}=\left(\sum_{k=0}^{n} \stackrel{k}{U}\right)^{\sim}=\sum_{k=0}^{n}(-1)^{k(k-1) / 2} \stackrel{k}{U}
$$

Отметим, что реверс обращает порядок следования множителей в произведениях генераторов: $\left(e^{a_{1}} e^{a_{2}} \ldots e^{a_{k}}\right)^{\sim}=e^{a_{k}} \ldots e^{a_{2}} e^{a_{1}}$, в частности $\left(e^{a}\right)^{\sim}=e^{a}$. Верны равенства $U^{\sim \sim}=U,(U V)^{\sim}=V^{\sim} U^{\sim},(U+V)^{\sim}=U^{\sim}+V^{\sim},(\lambda U)^{\sim}=\lambda U^{\sim}$.

Четностное сопряжение. Операция четностного сопряжения $U \rightarrow U^{\curlywedge}$ такова, что нечетные элементы она умножает на -1 , а четные элементы не меняет. В частности, имеем $\left(e^{a}\right)^{\curlywedge}=-e^{a}$. Для элемента $U \in \mathcal{C} \ell(p, q)$ имеем $U^{\curlywedge}=$ $\sum_{k=0}^{n}(-1)^{k} \stackrel{k}{U}$. Верны равенства $U^{\curlywedge \curlywedge}=U,(U V)^{\curlywedge}=U^{\curlywedge} V^{\curlywedge},(U+V)^{\curlywedge}=U^{\curlywedge}+V^{\curlywedge}$, $(\lambda U)^{\curlywedge}=\lambda U^{\curlywedge}$

Операции проецирования на подпространства $\mathcal{C} \ell_{k}^{\mathbb{F}}(p, q)$ и операция взятия следа. Введем обозначение для линейных операций проецирования на подпространства элементов ранга $k$ :

$$
\langle U\rangle_{k}=\stackrel{k}{U}=\sum_{a_{1}<\cdots<a_{k}} u_{a_{1} \ldots a_{k}} e^{a_{1} \ldots a_{k}} \in \mathcal{C} \ell_{k}^{\mathbb{F}}(p, q) .
$$

Введем операцию следа элемента $U \in \mathcal{C} \mathcal{C}^{\mathbb{F}}(p, q)$ как операцию проецирования на одномерное подпространство $\mathcal{C} \ell_{0}^{\mathbb{F}}(p, q)$, натянутое на единичный элемент $e: \operatorname{Tr}(U)=$ $\left.\langle U\rangle_{0}\right|_{e \rightarrow 1}=u$.

Будем обозначать коммутатор и антикоммутатор двух элементов алгебры Клиффорда через $[U, V]=U V-V U$ и $\{U, V\}=U V+V U$.

Следующая известная теорема говорит о центре алгебры Клиффорда $\mathcal{C} \ell^{\mathbb{F}}(p, q)$.

Теорема 1. Центром алгебры Клифборда $\mathcal{C}^{\mathbb{F}}(p, q)$ размерности $n=p+q$ является

$$
\operatorname{cen} \mathcal{C} \ell^{\mathbb{F}}(p, q)= \begin{cases}\mathcal{C} \ell_{0}^{\mathbb{F}}(p, q), & \text { n четное } \\ \mathcal{C} \ell_{0}^{\mathbb{E}}(p, q) \oplus \mathcal{C} \ell_{n}^{\mathbb{F}}(p, q), & n \text { нечетное. }\end{cases}
$$

Приведем также общеизвестные теоремы об изоморфизме алгебр Клиффорда матричным алгебрам. 
Теорема 2 (Периодичность Картана-Ботта). Вещественные алгебры Клифборда $\mathcal{C}^{\mathbb{R}}(p, q), n=p+q$, изоморфны (как алгебры) следующим матричным алгебрам:

$$
\mathcal{C} \ell^{\mathbb{R}}(p, q) \simeq \begin{cases}\operatorname{Mat}\left(2^{n / 2}, \mathbb{R}\right), & p-q \equiv 0,2 \quad(\bmod 8), \\ \operatorname{Mat}\left(2^{(n-1) / 2}, \mathbb{R}\right) \oplus \operatorname{Mat}\left(2^{(n-1) / 2}, \mathbb{R}\right), & p-q \equiv 1 \quad(\bmod 8), \\ \operatorname{Mat}\left(2^{(n-1) / 2}, \mathbb{C}\right), & p-q \equiv 3,7 \quad(\bmod 8), \\ \operatorname{Mat}\left(2^{(n-2) / 2}, \mathbb{H}\right), & p-q \equiv 4,6 \quad(\bmod 8), \\ \operatorname{Mat}\left(2^{(n-3) / 2}, \mathbb{H}\right) \oplus \operatorname{Mat}\left(2^{(n-3) / 2}, \mathbb{H}\right), & p-q \equiv 5 \quad(\bmod 8),\end{cases}
$$

где $\operatorname{Mat}(k, \mathbb{F})$ - алгебра квадратных матрии, размера $k \times k$ над полем или телом $\mathbb{F}$.

ТЕОрема 3. Имеем следующие изоморфизмы комплексных алгебр Клифборда матричным алгебрам:

$$
\mathcal{C} \ell(p, q) \simeq \begin{cases}\operatorname{Mat}\left(2^{n / 2}, \mathbb{C}\right), & n \text { четно }, \\ \operatorname{Mat}\left(2^{(n-1) / 2}, \mathbb{C}\right) \oplus \operatorname{Mat}\left(2^{(n-1) / 2}, \mathbb{C}\right), & n \text { нечетно }\end{cases}
$$

Отметим, что элементы комплексных алгебр Клиффорда $\mathcal{C}(p, q)$ представляются комплексными матрицами, размер которых равен $2^{[(n+1) / 2]}$, причем при нечетном $n$ это будут блочно-диагональные матрицы размера $2^{(n+1) / 2}$, у которых на диагонали стоят два блока размера $2^{(n-1) / 2}$, а остальные элементы - нули.

\section{3. ОБОБЩЕННАЯ ТЕОРЕМА ПАУЛИ}

В работе [20] автором были предложены теоремы, обобщающие так называемую фундаментальную теорему Паули о гамма-матрицах. При этом использовался аппарат алгебр Клиффорда. Сформулируем доказанные теоремы.

Будем обозначать через $\mathcal{I}$ множество мультииндексов $A$ длины от 0 до $n: \mathcal{I}=$ $\{\varnothing, 1, \ldots, n, 12,13, \ldots, 1, \ldots, n\}$, где $\varnothing-$ пустой мультииндекс. Пусть

$$
\mathcal{I}_{\text {even }}=\{A \in \mathcal{I}:|A| \text { четно }\}, \quad \mathcal{I}_{\text {odd }}=\{A \in \mathcal{I}:|A| \text { нечетно }\} .
$$

Используем обозначения $\gamma_{A}=\gamma_{a_{1} \ldots a_{k}}=\gamma_{a_{k}} \ldots \gamma_{a_{1}}=\left(\gamma^{A}\right)^{-1}$ и $\gamma_{a}=\eta^{a b} \gamma^{b}=\left(\gamma^{a}\right)^{-1}$.

Теорема 4. Пусть $\mathcal{C} \ell^{\mathbb{F}}(p, q)$ - вещественная (или комплексная) алгебра Клибборда четной размерности $n=p+q$. Пусть два набора элементов алгебры Клифборда $\gamma^{a}, \beta^{a}, a=1,2, \ldots, n$, удовлетворяют соотношениям

$$
\gamma^{a} \gamma^{b}+\gamma^{b} \gamma^{a}=2 \eta^{a b} e, \quad \beta^{a} \beta^{b}+\beta^{b} \beta^{a}=2 \eta^{a b} e .
$$

Тогда оба набора генерируют базисы ${ }^{4)}$ алгебры Клиффорда и существует единственный, с точностью до умножения на ненулевое вещественное (или комплексное) число, обратимый элемент алгебры Клифборда $T \in \mathcal{C} \ell^{\mathbb{F}}(p, q)$ такой, что

$$
\gamma^{a}=T^{-1} \beta^{a} T \quad \forall a=1, \ldots, n .
$$

При этом такой элемент $T$ имеет вид $T=\sum_{A} \beta^{A} F \gamma_{A}$, где в качестве элемента $F$ подойдет любой такой элемент из $\left\{\gamma^{A}, A \in \mathcal{I}_{\text {even }}\right\}$, если $\beta^{1 \ldots n} \neq-\gamma^{1 \ldots n}$, или из $\left\{\gamma^{A}, A \in \mathcal{I}_{\text {odd }}\right\}$, если $\beta^{1 \ldots n} \neq \gamma^{1 \ldots n}$, ито $\sum_{A} \beta^{A} F \gamma_{A} \neq 0$.

4) Другими словами, всевозможные произведения $\gamma^{a_{1}} \ldots \gamma^{a_{k}}=\gamma^{a_{1} \ldots a_{k}}=\gamma^{A}$ и $\beta^{A}$ образуют два базиса в $\mathcal{C} \ell^{\mathbb{F}}(p, q)$. 
ТЕОРема 5. Пусть $\mathcal{C} \ell^{\mathbb{R}}(p, q)$ - вещественная алгебра Клиффорда нечетной размерности $n=p+q$. Пусть два набора элементов алгебры Клифборда $\gamma^{a}, \beta^{a}$, $a=1,2, \ldots, n$, удовлетворяют соотношениям

$$
\gamma^{a} \gamma^{b}+\gamma^{b} \gamma^{a}=2 \eta^{a b} e, \quad \beta^{a} \beta^{b}+\beta^{b} \beta^{a}=2 \eta^{a b} e .
$$

Тогда в случае алгебры Клифборда $\mathcal{C} \ell^{\mathbb{R}}(p, q)$ сигнатуры $p-q \equiv 1(\bmod 4)$ элеменmъ $\gamma^{1 \ldots n}$ и $\beta^{1 \ldots n}$ либо принимают значения $\pm e^{1 \ldots n}$ и соответствующие наборь

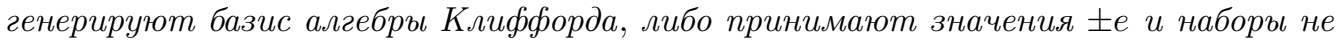
генерируют базис. При этом реализуются указанные ниже случаи 1-4.

В случае алгебрь Клифборда $\mathcal{C} \ell^{\mathbb{R}}(p, q)$ сигнатурь $p-q \equiv 3(\bmod 4)$ элементы $\gamma^{1 \ldots n}$ и $\beta^{1 \ldots n}$ всегда принимают значения $\pm e^{1 \ldots n}$ и соответствующие наборы всегда генерируют базис алгебры Клифборда. При этом реализуются только случаи 1, 2.

Утверждается, что существует единственный, с точностью до умножения на обратимый элемент центра алгебры Клифборда, обратимый элемент алгебры Клифборда T такой, что:

$$
\begin{array}{llll}
\text { 1) } & \gamma^{a}=T^{-1} \beta^{a} T & \forall a=1, \ldots, n & \Leftrightarrow \quad \beta^{1 \ldots n}=\gamma^{1 \ldots n} ; \\
\text { 2) } & \gamma^{a}=-T^{-1} \beta^{a} T & \forall a=1, \ldots, n \quad \Leftrightarrow \quad \beta^{1 \ldots n}=-\gamma^{1 \ldots n} ; \\
\text { 3) } \quad \gamma^{a}=e^{1 \ldots n} T^{-1} \beta^{a} T & \forall a=1, \ldots, n & \Leftrightarrow & \beta^{1 \ldots n}=e^{1 \ldots n} \gamma^{1 \ldots n} ; \\
\text { 4) } \quad \gamma^{a}=-e^{1 \ldots n} T^{-1} \beta^{a} T & \forall a=1, \ldots, n & \Leftrightarrow & \beta^{1 \ldots n}=-e^{1 \ldots n} \gamma^{1 \ldots n} .
\end{array}
$$

Заметим, что все четыре случая имеют единую запись в виде

$$
\gamma^{a}=\left(\beta^{1 \ldots n} \gamma_{1 \ldots n}\right) T^{-1} \beta^{a} T
$$

Кроме того, в случае вещественной алгебрь Клифборда $p-q \equiv 1(\bmod 4)$ элемент $T$, о существовании которого говорится во всех четырех случаях теоремы, имеет вид

$$
T=\sum_{A \in \mathcal{I}_{\text {even }}} \beta^{A} F \gamma_{A}
$$

где в качестве $F$ всегда подойдет один из элементов множества $\left\{\gamma^{A}+\gamma^{B}, A, B \in\right.$ $\left.\mathcal{I}_{\text {even }}\right\}$.

$B$ случае вещественной алгебры Клиффорда сигнатуры $p-q \equiv 3(\bmod 4)$ элемент $T$, о существовании которого говорится в случаях 1, 2 теоремь, имеет вид (5). Причем в качестве $F$ подойдет любой такой из элементов множества $\left\{\gamma^{A}, A \in \mathcal{I}_{\text {even }}\right\}$, что построенная по нему свертка $\sum_{A \in \mathcal{I}_{\text {even }}} \beta^{A} F \gamma_{A} \neq 0$.

Теорема 6. Пусть $\mathcal{C}(p, q)$ - комплексная алгебра Клиффорда нечетной размерности $n=p+q$. Пусть два набора элементов алгебры Клифборда $\gamma^{a}, \beta^{a}, a=$ $1,2, \ldots, n$, удовлетворяют соотношениям

$$
\gamma^{a} \gamma^{b}+\gamma^{b} \gamma^{a}=2 \eta^{a b} e, \quad \beta^{a} \beta^{b}+\beta^{b} \beta^{a}=2 \eta^{a b} e .
$$

Тогда в случае алгебры Клиффорда $\mathcal{C}(p, q)$ сигнатуры $p-q \equiv 1(\bmod 4)$ имеем для элементов $\gamma^{1 \ldots n}$ и $\beta^{1 \ldots n}$ возможные значения $\pm e^{1 \ldots n}$, когда соответствующие наборы генерируют базисы алгебры Клифборда, и значения $\pm е$, когда наборы не генерируют базис. При этом реализуются случаи 1-4 теоремы. 
$B$ алгебре Клиффорда $\mathcal{C}(p, q)$ сигнатуры $p-q \equiv 3(\bmod 4)$ имеем для элементов $\gamma^{1 \ldots n}$ и $\beta^{1 \ldots n}$ возможные значения $\pm i e^{1 \ldots n}$, когда соответствующие наборь генерируют базисы алгебры Клиффорда, и значения $\pm і е$, когда наборы не генерируют базис. При этом реализуются случаи 1, 2, 5, 6 теоремы.

Утверждается, что существует единственный, с точностъю до умножения на обратимый элемент иентра алгебры Клифборда, обратимый элемент алгебры Клифборда T такой, что:
1) $\gamma^{a}=T^{-1} \beta^{a} T$
$\forall a=1, \ldots, n \quad \Leftrightarrow \quad \beta^{1 \ldots n}=\gamma^{1 \ldots n} ;$
2) $\gamma^{a}=-T^{-1} \beta^{a} T$
$\forall a=1, \ldots, n \quad \Leftrightarrow \quad \beta^{1 \ldots n}=-\gamma^{1 \ldots n} ;$
3) $\gamma^{a}=e^{1 \ldots n} T^{-1} \beta^{a} T$
$\forall a=1, \ldots, n \quad \Leftrightarrow \quad \beta^{1 \ldots n}=e^{1 \ldots n} \gamma^{1 \ldots n} ;$
4) $\gamma^{a}=-e^{1 \ldots n} T^{-1} \beta^{a} T$
$\forall a=1, \ldots, n \quad \Leftrightarrow \quad \beta^{1 \ldots n}=-e^{1 \ldots n} \gamma^{1 \ldots n} ;$
5) $\gamma^{a}=i e^{1 \ldots n} T^{-1} \beta^{a} T$
$\forall a=1, \ldots, n \quad \Leftrightarrow \quad \beta^{1 \ldots n}=i e^{1 \ldots n} \gamma^{1 \ldots n} ;$
6) $\gamma^{a}=-i e^{1 \ldots n} T^{-1} \beta^{a} T$
$\forall a=1, \ldots, n \quad \Leftrightarrow \quad \beta^{1 \ldots n}=-i e^{1 \ldots n} \gamma^{1 \ldots n}$.

Заметим, что все шесть случаев имеют единую запись в виде

$$
\gamma^{a}=\left(\beta^{1 \ldots n} \gamma_{1 \ldots n}\right) T^{-1} \beta^{a} T
$$

Кроме того, элемент $T$, о существовании которого говорится во всех шести случаях теоремы, имеет вид

$$
\sum_{A \in \mathcal{I}_{\text {even }}} \beta^{A} F \gamma_{A}
$$

где в качестве $F$ всегда подойдет один из элементов множества $\left\{\gamma^{A}+\gamma^{B}, A, B \in\right.$ $\left.\mathcal{I}_{\text {even }}\right\}$.

Отметим, что предложенные теоремы можно переформулировать в терминах матриц, если воспользоваться утверждениями теорем 2, 3. В настоящем изложении делать этого не будем в силу громоздкости получающихся формулировок.

\section{4. СПИНОРЫ ДИРАКА И ВЕЙЛЯ В ФОРМАЛИЗМЕ АЛГЕБР КЛИФФОРДА}

Впервые спиноры были рассмотрены Картаном в 1913 году, затем переоткрыты Дираком в 1928 году. С тех пор был развит ряд подходов и предложен ряд различных реализаций спиноров, в том числе в случае произвольных размерностей пространства.

Открытие в 1928 году уравнения Дирака [27] привлекло внимание к изучению спиноров со стороны многих математиков и физиков. В 1930 году ЖКуве [31] и Заутер [32] рассматривали спинор как элемент левого идеала в матричной алгебре. В 1947 году Рисс [26] впервые интерпретировал спиноры как элементы левого идеала в алгебре Клиффорда. Именно такой подход наиболее удобен при рассмотрении $n$-мерных спиноров и изучении их свойств (см., например, [30], [33]). Упомянем также ставшие классическими работы Рашевского [34] и Румера [35].

Отметим, что понятие "спинор" включает в себя два аспекта. Первый аспект (алгебраический) заключается в том, что спинор (в простейшем случае) является элементом некоторого минимального левого идеала (см. ниже), т. е. фактически 
просто столбцом, если использовать матричный формализм. Второй аспект связан с тем, что на самом деле спинор представляет собой набор (столбец) функций, зависящих от точки пространства, и при ортогональных преобразованиях с матрицей $P \in \mathrm{O}(p, q)$ умножается слева на элемент спинорной группы. Дальнейшие рассмотрения в настоящей статье почти всегда будут затрагивать только первый, алгебраический аспект. Второй аспект следует принимать во внимание, когда мы рассматриваем спинор не просто как абстрактный алгебраический объект, а как неизвестную величину в уравнении Дирака.

Спиноры Дирака. Рассмотрим вещественную алгебру Клиффорда $\mathcal{C} \ell^{\mathbb{R}}(p, q)$ и примитивный идемпотент $t^{2}=t, t \in \mathcal{C} \ell^{\mathbb{R}}(p, q)$. Ему соответствует минимальный левый идеал ( спинорное пространство) $I(t)=\mathcal{C} \mathbb{R}^{\mathbb{R}}(p, q) t$, порожденный идемпотентом $t$. Элементы левого идеала $\psi \in I(t)$ будем называть спинорами (в формализме алгебр Клиффорда).

Неприводимое представление алгебры Клиффорда $\mathcal{C}(p, q) \rightarrow \operatorname{End}(I)$ инъективно в случае $p-q \neq 1(\bmod 4)($ см. теорему 2$)$. В случае $p-q \equiv 1(\bmod 4)$ алгебра Клиффорда не является простой и представляет собой прямую сумму двух простых идеалов. В этом случае рассматривается левый идеал (двойное спинорное пространcmвo) $I \oplus \hat{I}$, построенный по идемпотентам $t$ и $\hat{t}$. Таким образом, получаем инъективное представление в случае $p-q \equiv 1(\bmod 4)$. Однако оно является приводимым и представляет собой прямую сумму двух неприводимых представлений. Каждое из этих неприводимых представлений называется полуспинорным, и соответствующие левые идеалы называются полуспинорными пространствами.

При рассмотрении комплексной алгебры Клиффорда $\mathcal{C}(p, q)$ действуют аналогичным образом (см. теорему 3 ). В случае четного $n$ рассматривают спинорное представление. Элементы левого идеала называют спинорами Дирака. В случае нечетного $n$ рассматривают либо двойнъе спиноры, либо полуспиноры. Далее будем рассматривать только комплексные алгебры Клиффорда.

Спиноры Вейля. Рассмотрим множество спиноров Дирака, реализуемое в комплексной алгебре Клиффорда (как множество элементов левого идеала) $E_{\text {Dirac }}=$ $\{\psi \in I(t)\}$. Рассмотрим киральный оператор (или псевдоскаляр)

$$
\omega=\left\{\begin{array}{lll}
e^{1 \ldots n}, & p-q \equiv 0,1 \quad(\bmod 4), \\
i e^{1 \ldots n}, & p-q \equiv 2,3 \quad(\bmod 4) .
\end{array}\right.
$$

Нетрудно проверить, что $\omega=\omega^{-1}=\omega^{\dagger}$. Кроме того, $\left\{\omega, e^{a}\right\}=\omega e^{a}+e^{a} \omega=0$, если $n$ четно, и $\left[\omega, e^{a}\right]=\omega e^{a}-e^{a} \omega=0$, если $n$ нечетно, для всех $a=1,2, \ldots, n$.

Определим два оператора

$$
P_{\mathrm{L}}=\frac{e-\omega}{2}, \quad P_{\mathrm{R}}=\frac{e+\omega}{2} .
$$

Они являются ортогональными идемпотентами (проекторами), так как $\left(P_{\mathrm{R}}\right)^{2}=P_{\mathrm{R}}$, $\left(P_{\mathrm{L}}\right)^{2}=P_{\mathrm{L}}, P_{\mathrm{R}} P_{\mathrm{L}}=P_{\mathrm{L}} P_{\mathrm{R}}=0$. Отметим, что в случае нечетного $n$ операторы $P_{\mathrm{L}}$ и $P_{\mathrm{R}}$ лежат в центре алгебры Клиффорда $\mathcal{C}(p, q)$ и дают разложение алгебры Клиффорда на прямую сумму двух идеалов $\mathcal{C}(p, q)=P_{\mathrm{R}} \mathcal{C}(p, q) \oplus P_{\mathrm{L}} \mathcal{C}(p, q)$. 
Рассмотрим комплексную алгебру Клиффорда $\mathcal{C}(p, q)$ четной размерности $n=$ $p+q$. Тогда левые и правые спиноры Вейля (или киралъные спиноры) определяются следующим образом:

$$
E_{\text {Weyl }}^{\mathrm{L}}=\left\{\psi \in E_{\text {Dirac }} \mid P_{\mathrm{L}} \psi=\psi\right\}, \quad E_{\text {Weyl }}^{\mathrm{R}}=\left\{\psi \in E_{\text {Dirac }} \mid P_{\mathrm{R}} \psi=\psi\right\} .
$$

Таким образом, спиноры Вейля являются собственными спинорами операторов $P_{\mathrm{L}}$ и $P_{\mathrm{R}}$. Заметим, что условия на спиноры можно переписать в другом эквивалентном виде: $P_{\mathrm{L}} \psi=\psi \Leftrightarrow \omega \psi=-\psi$ и $P_{\mathrm{R}} \psi=\psi \Leftrightarrow \omega \psi=\psi$. Отметим, что $E_{\text {Dirac }}=$

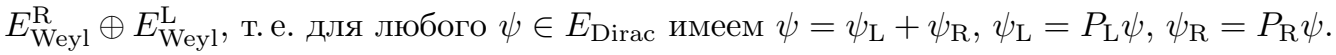

\section{5. СОГЛАСОВАННОСТЬ МАТРИЧНЫХ ОПЕРАЦИЙ И ОПЕРАЦИЙ В АЛГЕБРАХ КЛИФФОРДА}

Будем рассматривать матричные представления комплексных алгебр Клиффорда (см. теорему 2)

$$
\gamma: \mathcal{C}(p, q) \rightarrow \begin{cases}\operatorname{Mat}\left(2^{n / 2}, \mathbb{C}\right), & n \text { четное } \\ \operatorname{Mat}\left(2^{(n-1) / 2}, \mathbb{C}\right) \oplus \operatorname{Mat}\left(2^{(n-1) / 2}, \mathbb{C}\right), & n \text { нечетное. }\end{cases}
$$

В случае нечетного $n$ элементы алгебры Клиффорда представляются блочно-диагональными матрицами размера $2^{(n+1) / 2}$. Обозначим через $\gamma^{a}=\gamma\left(e^{a}\right)$ матрицы, соответствующие генераторам алгебры Клиффорда в матричном представлении $\gamma$.

Согласно теореме Паули все остальные матричные представления $\beta$ в случае четного $n$ можно получить из исходного в виде $\beta^{a}=T^{-1} \gamma^{a} T$, где обратимая матрица $T$ определена с точностью до ненулевой константы.

В случае нечетного $n$ все матричные представления можно получить из исходного в виде $\beta^{a}= \pm T^{-1} \gamma^{a} T$, где матрица $T$ определена с точностью до умножения на обратимый элемент центра $Z=\lambda \mathbf{1}+\mu J$, где $J=\operatorname{diag}(1,1, \ldots, 1,-1, \ldots,-1)-$ диагональная матрица, у которой на диагонали стоит одинаковое число 1 и -1 .

Рассмотрим операцию эрмитова сопряжения от элемента алгебры Клиффорда, которая вводится по формулам (см. [36])

$$
\begin{aligned}
U^{\dagger} & = \begin{cases}e_{1 \ldots p} U^{\ddagger} e^{1 \ldots p}, & p \text { нечетно, } \\
e_{1 \ldots p} U^{\ddagger \curlywedge} e^{1 \ldots p}, & p \text { четно, }\end{cases} \\
U^{\dagger} & = \begin{cases}e_{p+1 \ldots n} U^{\ddagger} e^{p+1 \ldots n}, & q \text { четно, } \\
e_{p+1 \ldots n} U^{\ddagger \curlywedge} e^{p+1 \ldots n}, & q \text { нечетно. }\end{cases}
\end{aligned}
$$

Всегда можно выбрать такое матричное представление $\gamma\left(\right.$ см. [36]), что $\gamma\left(e^{a}\right)=$ $\gamma^{a}$ - унитарные матрицы:

$$
\left(\gamma^{a}\right)^{\dagger}=\left(\gamma^{a}\right)^{-1}=\eta^{a a} \gamma^{a}
$$

В дальнейшем будем рассматривать только матричные представления, для которых выполнено (8). Заметим, что соотношение (8) эквивалентно $\left(\gamma\left(e^{a}\right)\right)^{\dagger}=\gamma\left(\left(e^{a}\right)^{\dagger}\right)$, а значит, эквивалентно $\gamma^{\dagger}(U)=\gamma\left(U^{\dagger}\right) \forall U \in \mathcal{C}(p, q)$, т. е. согласованности операции эрмитова сопряжения от матрицы и операции эрмитова сопряжения от элемента 
алгебры Клиффорда. Также заметим, что из (8) следует, что первые $p$ матриц $\gamma^{a}$ являются эрмитовыми, а последние $q$ - антиэрмитовыми.

Будем рассматривать различные операции сопряжения (см. раздел 2) от элементов алгебры Клиффорда $\mathcal{C}(p, q): U^{\sim}, U^{\curlywedge}, \bar{U}, U^{\ddagger}, U^{\dagger}, U^{\mathrm{T}}, \overleftarrow{U}$.

Мы рассматриваем здесь две новые операции от элементов алгебры Клиффорда: операцию транспонирования элемента алгебры Клифборда и операчию взятия комплексного матричного сопряжения от элемента алгебры Клифборда

$$
U^{\mathrm{T}}=\gamma^{-1}\left((\gamma(U))^{\mathrm{T}}\right), \quad \overleftarrow{U}=\gamma^{-1}(\overleftarrow{\gamma(U)})
$$

которые зависят от выбора матричного представления $\gamma$. Здесь $\overleftarrow{\gamma(U)}-$ матрица, комплексно-сопряженная к матрице $\gamma(U)$.

Рассмотрим набор из генераторов $e^{a}$ алгебры Клиффорда $\mathcal{C}(p, q)$. Отметим, что $\left(e^{a}\right)^{\sim}=e^{a},\left(e^{a}\right)^{\curlywedge}=-e^{a}, \overline{e^{a}}=e^{a},\left(e^{a}\right)^{\ddagger}=e^{a}$. Будем рассматривать наборы элементов, полученные из исходного набора $e^{a}$ действием одной из операций сопряжения:

$$
\beta^{a}= \pm\left(e^{a}\right)^{\dagger}, \quad \pm\left(e^{a}\right)^{\mathrm{T}}, \quad \pm \overleftarrow{e^{a}}
$$

Заметим, что все рассмотренные наборы удовлетворяют определяющим соотношениям алгебры Клиффорда $\beta^{a} \beta^{b}+\beta^{b} \beta^{a}=2 \eta^{a b} e$.

Справедлива следующая теорема.

Теорема 7. Наборы $\beta^{a}$, введенные в (10), генерируют новые базисы алгебры Клифборда $\mathcal{C} \ell(p, q)$.

В случае четного $n$ для рассматриваемых наборов $e^{a}$ и $\beta^{a}$ всегда существует единственный, с точностью до умножения на комплексную константу, обратимый элемент $T$ такой, что

$$
\beta^{a}=T^{-1} e^{a} T, \quad a=1, \ldots, n .
$$

При этом $T \in \mathcal{C} \ell_{\text {even }}(p, q)$, если $\beta^{1 \ldots n}=e^{1 \ldots n}, u T \in \mathcal{C} \ell_{\text {odd }}(p, q)$, ecли $\beta^{1 \ldots n}=-e^{1 \ldots n}$.

Элемент $T$ имеет вид $\sum_{A} \beta^{A} e^{B} e_{A}$ для некоторого $e^{B}$.

$B$ случае нечетного $n$ для рассматриваемьх двух наборов $e^{a}$ и $\beta^{a}$ существует единственный, с точностъю до умножения на обратимый элемент иентра, обратимый элемент $T$ такой, что

$$
\begin{array}{lll}
\beta^{a}=T^{-1} e^{a} T, & a=1, \ldots, n & \left(\text { в случае } \beta^{1 \ldots n}=e^{1 \ldots n}\right), \\
\beta^{a}=-T^{-1} e^{a} T, & a=1, \ldots, n & \left(\text { в случае } \beta^{1 \ldots n}=-e^{1 \ldots n}\right) .
\end{array}
$$

В обоих случаях элемент $T \in \mathcal{C l}_{\mathrm{even}}(p, q)$ (либо после умножения на $e^{1 \ldots n}$ получаем другой $\left.T \in \mathcal{C l}_{\text {odd }}(p, q)\right)$.

Кроме того, элемент $T$ имеет вид $\sum_{A \in \mathcal{I}_{\text {even }}} \beta^{A} e^{B} e_{A}$ для некоторого $e^{B}$.

ДокАЗАТЕЛЬСтво теоремы следует из теорем 4-6. 


\section{6. ДОПОЛНИТЕЛЬНЫЕ СИГНАТУРЫ АЛГЕБР КЛИФФОРДА}

Заметим, что можно всегда выбрать такие матричные представления $\gamma$, для которых помимо условий (8) выполнены условия

$$
\left(\gamma^{a}\right)^{\mathrm{T}}= \pm \gamma^{a}, \quad \overleftarrow{\gamma^{a}}= \pm \gamma^{a}
$$

т. е. матрицы $\gamma^{a}$ являются вещественными или чисто мнимыми (и при этом симметричными или антисимметричными в силу унитарности).

Введем дополнительные сигнатуры алгебры Клифборда

$$
\begin{array}{ll}
\left(k_{\gamma}, l_{\gamma}\right), & 0 \leqslant k_{\gamma} \leqslant n, \quad 0 \leqslant l_{\gamma} \leqslant n, \quad k_{\gamma}+l_{\gamma}=n, \\
\left(r_{\gamma}, s_{\gamma}\right), & 0 \leqslant r_{\gamma} \leqslant n, \quad 0 \leqslant s_{\gamma} \leqslant n, \quad r_{\gamma}+s_{\gamma}=n,
\end{array}
$$

которые зависят от матричного представления $\gamma$ алгебры Клиффорда. Здесь $k_{\gamma}-$ количество симметричных матриц среди $\gamma^{a}, l_{\gamma}$ - количество антисимметричных матриц среди $\gamma^{a}$. Аналогично $r_{\gamma}$ - количество вещественных матриц среди $\gamma^{a}, s_{\gamma}-$ количество чисто мнимых матриц среди $\gamma^{a}$.

Введенные величины зависят от матричного представления $\gamma$. Далее будем иногда опускать индекс $\gamma$. Заметим, что, несмотря на зависимость от матричного представления, мы найдем ограничения на возможные значения параметров $k_{\gamma}$ и $l_{\gamma}$ (см. ниже теорему 12).

Параметров $k, l, r, s$ порой не хватает, чтобы полностью восстановить нужную нам информацию о выбранном матричном представлении алгебры Клиффорда. Поэтому мы предлагаем начать с двух других независимых параметров $[k p]_{\gamma},[k q]_{\gamma}$, которые равны соответственно количеству симметричных матриц среди первых $p$ матриц $\gamma^{a}$ и количеству симметричных матриц среди последних $q$ матриц $\gamma^{a}$. Заметим, что $[k p]_{\gamma} \leqslant k,[k p]_{\gamma} \leqslant p,[k q]_{\gamma} \leqslant k,[k q]_{\gamma} \leqslant q$. Этих двух параметров (наряду с фиксированными и не зависящими от матричного представления параметрами $p$ и $q)$ нам хватит, чтобы однозначно восстановить параметры $k_{\gamma}, l_{\gamma}, r_{\gamma}, s_{\gamma},[l p]_{\gamma},[l q]_{\gamma}$. Последние два из перечисленных параметров равны соответственно количеству антисимметричных матриц среди первых $p$ матриц $\gamma^{a}$ и количеству антисимметричных матриц среди последних $q$ матриц $\gamma^{a}$.

Имеем следующие формулы для этих параметров:

$$
\begin{gathered}
{[l p]_{\gamma}=p-[k p]_{\gamma}, \quad[l q]_{\gamma}=q-[k q]_{\gamma}} \\
k_{\gamma}=[k p]_{\gamma}+[k q]_{\gamma}, \quad l_{\gamma}=[l p]_{\gamma}+[l q]_{\gamma}=p+q-[k p]_{\gamma}-[k q]_{\gamma}=n-k, \\
r_{\gamma}=[k p]_{\gamma}+[l q]_{\gamma}=[k p]_{\gamma}+q-[k q]_{\gamma}, \quad s_{\gamma}=[l p]_{\gamma}+[k q]_{\gamma}=p-[k p]_{\gamma}+[k q]_{\gamma} .
\end{gathered}
$$

Итак, в итоге размерность алгебры Клиффорда $n$ представляется суммой четырех чисел:

$$
n=[k p r]_{\gamma}+[l p s]_{\gamma}+[k q s]_{\gamma}+[l q r]_{\gamma},
$$

где $[k p r]_{\gamma}=[k p]_{\gamma},[l p s]_{\gamma}=[l p]_{\gamma},[k q s]_{\gamma}=[k q]_{\gamma},[l q r]_{\gamma}=[l q]_{\gamma}$. Здесь индексы $r$ и $s$ характеризуют вещественность или мнимость соответствующих матриц $\gamma^{a}$ (которая автоматически следует из свойств унитарности и (анти)симметричности). 
Например, в случае $(p, q)=(n, 0)$ имеем $[k q]_{\gamma}=[l q]_{\gamma}=0, k_{\gamma}=r_{\gamma}=[k p]_{\gamma}, l_{\gamma}=$ $s_{\gamma}=[l p]_{\gamma}=p-[k p]_{\gamma}$, т. е. в этом случае все симметричные матрицы, представляющие генераторы, являются вещественными, а все антисимметричные - мнимыми.

В случае $(p, q)=(0, n)$ имеем $[k p]_{\gamma}=[l p]_{\gamma}=0, k_{\gamma}=s_{\gamma}=[k q]_{\gamma}, l_{\gamma}=r_{\gamma}=[l q]_{\gamma}=$ $q-[k q]_{\gamma}$, т. е. все симметричные матрицы, представляющие генераторы, являются мнимыми, а все антисимметричные - вещественными.

При сделанных предположениях имеем для всех наборов (10) $\beta^{a}= \pm e^{a}$. Тогда согласно ОТП элементы $T$, о которых говорилось выше, найдутся среди элементов вида $\sum e^{A} e^{B} e_{A}$, т. е. среди элементов базиса $\left\{e^{A}\right\}$.

Обозначим те генераторы $e^{a}$, для которых матрицы $\gamma^{a}$ симметричны, через $e^{b_{1}}, \ldots$ $\ldots, e^{b_{k}}$. Аналогично для антисимметричных матриц имеем обозначение $e^{c_{1}}, \ldots, e^{c_{l}}$, для вещественных $-e^{d_{1}}, \ldots, e^{d_{r}}$ и для чисто мнимых $-e^{f_{1}}, \ldots, e^{f_{s}}$.

Можно указать явные формулы для операций $U^{\mathrm{T}}$ и $\overleftarrow{U}$. А именно, имеем следующий результат.

Теорема 8. Рассмотрим комплексную алгебру Клифборда $\mathcal{C}(p, q)$ и матричное представление $\gamma$, удовлетворяющее условиям (8), (11). Тогда верны следующие бормулы для операций транспонирования и взятия комплексного матричного сопряжения (9) от элемента алгебры Клифборда:

$$
\begin{aligned}
& U^{\mathrm{T}}= \begin{cases}e_{b_{1} \ldots b_{k}} U^{\sim} e^{b_{1} \ldots b_{k}}, & k \text { нечетно, } \\
e_{b_{1} \ldots b_{k}} U^{\sim \curlywedge} e^{b_{1} \ldots b_{k}}, & k \text { четно, }\end{cases} \\
& U^{\mathrm{T}}= \begin{cases}e_{c_{1} \ldots c_{l}} U^{\sim} e^{c_{1} \ldots c_{l}}, & l \text { четно, } \\
e_{c_{1} \ldots c_{l}} U^{\sim \curlywedge} e^{c_{1} \ldots c_{l}}, & l \text { нечетно, }\end{cases} \\
& \overleftarrow{U}= \begin{cases}e_{d_{1} \ldots d_{r}} \bar{U} e^{d_{1} \ldots d_{r}}, & r \text { нечетно }, \\
e_{d_{1} \ldots d_{r}} \overline{U^{\curlywedge}} e^{d_{1} \ldots d_{r}}, & r \text { четно },\end{cases} \\
& \overleftarrow{U}= \begin{cases}e_{f_{1} \ldots f_{s}} \bar{U} e^{f_{1} \ldots f_{s}}, & s \text { четно } \\
e_{f_{1} \ldots f_{s}} \overline{U \curlywedge} e^{f_{1} \ldots f_{s}}, & s \text { нечетно }\end{cases}
\end{aligned}
$$

ДокАЗАТЕЛЬСтво аналогично доказательству формул (7) (см. [36]).

Заметим, что формулы (12), (13) похожи на формулы для эрмитова сопряжения (7), однако они явно зависят от матричного представления. В зависимости от $k, l, r$ и $s$ всегда имеются по две эквивалентные формулы для операции транспонирования и по две эквивалентные формулы для операции взятия комплексного матричного сопряжения.

\section{7. ОБОБЩЕНИЕ ДИРАКОВСКОГО СОПРЯЖЕНИЯ}

В настоящем разделе введем аналоги дираковского сопряжения на случай произвольных размерности и сигнатуры пространства. При этом в случае четных размерностей $n$ будем рассматривать одновременно два различных дираковских сопряжения. В случае нечетного $n$ рассматривается только одно дираковское сопряжение.

Рассмотрим комплексную алгебру Клиффорда $\mathcal{C}(p, q)$. Согласно ОТП (теорема 7 ) и используя рассуждения из раздела 6 , заключаем, что среди элементов базиса 
$\left\{e^{A}\right\}$ найдутся элементы $A_{ \pm}$такие, что

$$
\left(e^{a}\right)^{\dagger}= \pm A_{ \pm}^{-1} e^{a} A_{ \pm}
$$

причем в случае четного $n$ всегда существуют оба элемента $A_{ \pm}$, а в случае нечетного $n$ - только один из элементов $A_{+}$и $A_{-}$. Эти формулы можно переписать в виде $U^{\dagger}=A_{+}^{-1} U^{\ddagger} A_{+}$и $U^{\dagger}=A_{-}^{-1} U^{\ddagger \curlywedge} A_{-}$. В следующей теореме представлены явные формулы для элементов $A_{ \pm}$. Отметим, что в литературе при четном $n$ в качестве элемента, осуществляющего дираковское сопряжение, используют элемент $e^{1 \ldots p}$. В следующей теореме разобраны всевозможные случаи.

Теорема 9. Элемент $A_{+}$существует всегда, кроме случая четного $р$ и нечетного $q$, а элемент $A_{-}$существует всегда, кроме случая нечетного $p$ и четного $q$. Для этих элементов верны формуль

$$
\begin{aligned}
& A_{+}= \begin{cases}\mu_{1} e^{1 \ldots p}, & p, q \text { нечетны }, \\
\mu_{2} e^{p+1 \ldots n}, & p, q \text { четны }, \\
Z_{1} e^{1 \ldots p}=Z_{2} e^{p+1 \ldots n}, & p \text { нечетно, } q \text { четно, }\end{cases} \\
& A_{-}= \begin{cases}\mu_{1} e^{p+1 \ldots n}, & p, q \text { нечетны, } \\
\mu_{2} e^{1 \ldots p}, & p, q \text { четны }, \\
Z_{1} e^{1 \ldots p}=Z_{2} e^{p+1 \ldots n}, & \text { p четно, } q \text { нечетно },\end{cases}
\end{aligned}
$$

где $\mu_{i}$ - произвольные ненулевые комплексные константы, а $Z_{i}$ - произвольные обратимые элементы центра алгебры Клифборда $\mathcal{C}(p, q)$.

Кроме того, всегда можно подобрать $\mu_{i} u Z_{i}$ так, чтобъ для элементов $A_{+}$ и $A_{-}$выполнялись соотношения

$$
A_{+}=A_{+}^{\dagger}=A_{+}^{-1}=A_{+}^{\ddagger}, \quad A_{-}=A_{-}^{\dagger}=A_{-}^{-1}=A_{-}^{\ddagger \curlywedge} .
$$

Доказательство. Согласно теореме 7 элемент $A_{+}$существует, если $\left(e^{1}\right)^{\dagger} \ldots$ $\ldots\left(e^{n}\right)^{\dagger}=e^{1 \ldots n}$, т. е. когда $q$ четно, а элемент $A_{-}$существует, если $\left(e^{1}\right)^{\dagger} \ldots\left(e^{n}\right)^{\dagger}=$ $-e^{1 \ldots n}$, т. е. когда $q$ нечетно. Явные выражения для элементов $A_{+}$и $A_{-}$получаем из (7). Выбирая в качестве $\mu$ число 1 или $i$ в зависимости от $p$ и $q$, можем добиться выполнения свойств (17).

В случае различных сигнатур можно ввести следующие операции сопряжения Дирака:

$$
\begin{array}{ll}
\psi^{\mathrm{D}_{+}}=\psi^{\dagger}\left(A_{+}\right)^{-1}=\left(A_{+}\right)^{-1} \psi^{\ddagger}, & \text { кроме случая } p \text { четно, } q \text { нечетно, } \\
\psi^{\mathrm{D}_{-}}=\psi^{\dagger}\left(A_{-}\right)^{-1}=\left(A_{-}\right)^{-1} \psi^{\ddagger \curlywedge}, & \text { кроме случая } p \text { нечетно, } q \text { четно. }
\end{array}
$$

Например, в случае алгебры Клиффорда $\mathcal{C}(1,3)$ с генераторами $e^{0}, e^{1}, e^{2}, e^{3}$ получаем два сопряжения Дирака $\psi^{\mathrm{D}_{+}}=\psi^{\dagger} e^{0}$ и $\psi^{\mathrm{D}_{-}}=\psi^{\dagger} e^{123}$, первое из которых совпадает со стандартным.

Рассмотрим следующие два набора величин, называемые билинейными ковариантами (билинейными формами Дирака): $j_{ \pm}^{A}=\psi^{\mathrm{D}_{ \pm}} e^{A} \psi$, где $A$ - произвольный мультииндекс длины от 0 до $n$. Можно проверить, что при ортогональных заменах 
координат $x^{\mu} \rightarrow x^{\prime \mu}=p_{\nu}^{\mu} x^{\nu}$, где $P=\left\|p_{\nu}^{\mu}\right\| \in \mathrm{O}(p, q)$, билинейные коварианты меняются (если спиноры меняются по правилу $\psi \rightarrow S \psi$, где $S$ - элемент спинорной группы) как тензоры либо почти как тензоры (в формуле преобразований появляется знак минус): $\left(j_{ \pm}^{\mu_{1} \ldots \mu_{k}}\right)^{\prime}=p_{\nu_{1}}^{\mu_{1}} \ldots p_{\nu_{k}}^{\mu_{k}} j_{ \pm}^{\nu_{1} \ldots \nu_{k}}$ или $\left(j_{ \pm}^{\mu_{1} \ldots \mu_{k}}\right)^{\prime}=-p_{\nu_{1}}^{\mu_{1}} \ldots p_{\nu_{k}}^{\mu_{k}} j_{ \pm}^{\nu_{1} \ldots \nu_{k}}$ в зависимости от $k$.

\section{8. ОБОБЩЕНИЕ МАЙОРАНОВСКОГО СОПРЯЖЕНИЯ И ТЕОРЕМА О ДОПОЛНИТЕЛЬНОЙ СИГНАТУРЕ АЛГЕБРЫ КЛИФФОРДА}

Рассмотрим комплексную алгебру Клиффорда $\mathcal{C}(p, q)$ и операцию транспонирования элемента алгебры Клиффорда (9). Заметим, что данная операция зависит от выбора матричного представления.

Согласно ОТП найдутся элементы $C_{ \pm}$такие, что

$$
\left(e^{a}\right)^{\mathrm{T}}= \pm C_{ \pm}^{-1} e^{a} C_{ \pm},
$$

причем в случае четного $n$ всегда существуют оба элемента $C_{ \pm}$, а в случае нечетного $n$ - только один из элементов $C_{+}$и $C_{-}$. Также можно переписать формулы в виде $U^{\mathrm{T}}=C_{+}^{-1} U^{\sim} C_{+}$и $U^{\mathrm{T}}=C_{-}^{-1} U^{\sim \curlywedge} C_{-}$.

Сформулируем следующие теоремы об элементах $C_{ \pm}$. Формулы (21) и (22) общеизвестны. Мы представляем также явный вид элементов $C_{ \pm}$(формулы $(23),(24)$ ) и выражения для констант $\lambda_{ \pm}$в зависимости от значений дополнительной сигнатуры $(k, l)$ (см. (25), (26)).

Теорема 10. Элемент $C_{+}$существует в случаях $n \neq 3(\bmod 4)$, элемент $C_{-}$ существует в случалх $n \neq 1(\bmod 4)$. Для этих элементов верны формулы

$$
\left(C_{ \pm}\right)^{\mathrm{T}}=\lambda_{ \pm} C_{ \pm}, \quad \overleftarrow{C_{ \pm}} C_{ \pm}=\lambda_{ \pm} e
$$

где

$$
\lambda_{+}=\left\{\begin{array}{lll}
+1, & n \equiv 0,1,2 \quad(\bmod 8), \\
-1, & n \equiv 4,5,6 \quad(\bmod 8),
\end{array} \quad \lambda_{-}=\left\{\begin{array}{lll}
+1, & n \equiv 0,6,7 & (\bmod 8), \\
-1, & n \equiv 2,3,4 & (\bmod 8) .
\end{array}\right.\right.
$$

Теорема 11. В случае матричных представлений, удовлетворяющих (8), (11), имеем явный вид элементов $C_{ \pm}$:

$$
\begin{aligned}
C_{+} & = \begin{cases}\mu_{1} e^{b_{1} \ldots b_{k}}, & k, l \text { нечетны, }, \\
\mu_{2} e^{c_{1} \ldots c_{l}}, & k, l \text { четны }, \\
Z_{1} e^{b_{l} \ldots b_{k}}=Z_{2} e^{c_{1} \ldots c_{l}}, & k \text { нечетно, } l \text { четно, }\end{cases} \\
C_{-} & = \begin{cases}\mu_{1} e^{c_{1} \ldots c_{l}}, & k, l \text { нечетны }, \\
\mu_{2} e^{b_{1} \ldots b_{k}}, & k, l \text { четнь }, \\
Z_{1} e^{b_{l} \ldots b_{k}}=Z_{2} e^{c_{1} \ldots c_{l}}, & k \text { четно, }, \text { нечетно },\end{cases}
\end{aligned}
$$

где $\mu_{i}$ - произвольные ненулевые комплексные константы, а $Z_{i}$ - обратимые элементы центра алгебры Клифборда $\mathcal{C}(p, q)$. Такэе получаем следующие значения 
$\partial л я \lambda_{ \pm}:$

$$
\begin{array}{r}
\lambda_{+}= \begin{cases}(-1)^{k(k-1) / 2}, & k, l \text { нечетны }, \\
(-1)^{l(l+1) / 2}, & k, l \text { четны }, \\
(-1)^{k(k-1) / 2}=(-1)^{l(l+1) / 2}, & k \text { нечетно, } l \text { четно },\end{cases} \\
\lambda_{-}= \begin{cases}(-1)^{l(l+1) / 2}, & k, l \text { нечетны }, \\
(-1)^{k(k-1) / 2}, & k, l \text { четны, } \\
(-1)^{k(k-1) / 2}=(-1)^{l(l+1) / 2}, & k \text { четно, }, \text { нечетно. }\end{cases}
\end{array}
$$

ДоказАТЕЛЬСтво теорем 10, 11. Явные формулы для элементов $C_{ \pm}$в случае выделенных матричных представлений получаем из формул (12).

Для четного $n$ из (20) получаем $e^{a}=C_{ \pm}^{\mathrm{T}} C_{ \pm}^{-1} e^{a} C_{ \pm}\left(C_{ \pm}^{-1}\right)^{\mathrm{T}}$, т. е. $C_{ \pm}\left(C_{ \pm}^{-1}\right)^{\mathrm{T}}=$ $\lambda_{ \pm} e$, так как полученное выражение коммутирует со всеми элементами. Поскольку $\operatorname{Det}\left(C_{ \pm}\right)=1$ (под определителем от элемента алгебры Клиффорда понимается определитель от соответствующего матричного представления, см. [37]), то $\lambda_{ \pm}=1,-1$. Кроме того, $C_{ \pm}^{\dagger} C_{ \pm}=e$, тогда получим $\overleftarrow{C_{ \pm}}=\left(C_{ \pm}^{\mathrm{T}}\right)^{\dagger}=\left(\lambda_{ \pm} C_{ \pm}\right)^{\dagger}=\lambda_{ \pm} C_{ \pm}^{-1}$

Далее, для произвольного упорядоченного мультииндекса $A$ длины $k$ имеем

$$
\begin{aligned}
\left(e^{A}\right)^{\mathrm{T}} & =( \pm 1)^{k}(-1)^{k(k-1) / 2} C_{ \pm}^{-1} e^{A} C_{ \pm}, \\
\left(C_{ \pm} e^{A}\right)^{\mathrm{T}} & =( \pm 1)^{k}(-1)^{k(k-1) / 2} \lambda_{ \pm}\left(C_{ \pm} e^{A}\right) .
\end{aligned}
$$

Заметим, что поскольку набор $e^{A}$ образует базис в алгебре Клиффорда, то и набор из симметричных и антисимметричных матриц $C_{ \pm} e^{A}$ также образует базис. Элементы представляются комплексными квадратными матрицами размера $2^{n / 2}$. Тогда среди них антисимметричных должно быть $(1 / 2) 2^{n / 2}\left(2^{n / 2}-1\right)$. С другой стороны, их число равно

$$
\sum_{k=0}^{n} \frac{1}{2}\left(1-( \pm 1)^{k}(-1)^{k(k-1) / 2} \lambda_{ \pm}\right) C_{n}^{k}
$$

Приравнивая два выражения, получаем

$$
\lambda_{ \pm}=\cos \frac{\pi n}{4} \pm \sin \frac{\pi n}{4}= \pm \sqrt{2} \sin \left(\frac{\pi}{4}(n \pm 1)\right)
$$

что и требовалось доказать.

Для нечетного $n$ можно получить те же формулы (21), если воспользоваться тем, что в случае выделенных матричных представлений элементы $C_{ \pm}$всегда найдутся среди элементов базиса $e^{A}$ (см. формулы (23) и (24)). Формулы (25) и (26) (для случая четного $n$ тоже) получаем, сопоставляя формулы (23), (24) и (21). Далее, выбрав какое-нибудь конкретное матричное представление (см., например, [36]), получаем значения $\lambda_{ \pm}$в зависимости от $n(\bmod 8)$ в случае нечетного $n$.

Заметим, что несмотря на то что элементы $C_{ \pm}$зависят от матричного представления, их симметричность (21) определяется только размерностью пространства $n(22)$. 
ТАБЛИЦА 1

\begin{tabular}{|c|c|c|c|}
\hline$n(\bmod 8)$ & $(k(\bmod 4), l(\bmod 4))$ & $n(\bmod 8)$ & $(k(\bmod 4), l(\bmod 4))$ \\
\hline \hline 0 & $(0,0),(1,3)$ & 4 & $(3,1),(2,2)$ \\
\hline 1 & $(1,0)$ & 5 & $(3,2)$ \\
\hline 2 & $(1,1),(2,0)$ & 6 & $(3,3),(0,2)$ \\
\hline 3 & $(2,1)$ & 7 & $(0,3)$ \\
\hline
\end{tabular}

Представим следующий новый результат. Несмотря на то что параметры $k$ и $l$ дополнительной сигнатуры (см. раздел 6) определяются выбором матричного представления, их значения не могут быть произвольными и принимают вполне определенные значения в зависимости от размерности пространства $n$.

Теорема 12. Рассмотрим комплексные алгебры Клифборда $\mathcal{C}(p, q)$ и матричное представление, удовлетворяющее условиям (8), (11). Тогда в зависимости от размерности $n=p+q$ алгебры Клифборда имеем возможные значения для дополнительной сигнатуры $\left(k_{\gamma}, l_{\gamma}\right)$, приведенные в табл. 1.

ДокАзАтЕЛьство. Доказательство проводится сопоставлением явных формул $(25),(26)$ для $\lambda_{ \pm}$, зависящих от $k$ и $l$, и формул $(22)$, зависящих от $n(\bmod 8)$.

Рассмотрим в качестве примера алгебры Клиффорда $\mathcal{C}(p, q)$ размерности $n=2$ и разных сигнатур $(p, q)=(2,0),(1,1),(0,2)$. Следующие три матрицы Паули антикоммутируют и в квадрате дают $+\mathbf{1}$ :

$$
\left(\begin{array}{cc}
0 & -i \\
i & 0
\end{array}\right), \quad\left(\begin{array}{ll}
0 & 1 \\
1 & 0
\end{array}\right), \quad\left(\begin{array}{cc}
1 & 0 \\
0 & -1
\end{array}\right) .
$$

В качестве $\gamma^{1}$ и $\gamma^{2}$ мы можем взять любые две из этих трех матриц (в случае необходимости домножая на мнимую единицу $i$ ). Таким образом, реализуются в точности только сигнатуры $(k, l)=(2,0),(1,1)$. Возможные значения дополнительных сигнатур для алгебр Клиффорда размерностей $n=1,2,3,4$ приведены в табл. 2.

Укажем алгоритм, по которому мы составили эту таблицу, пользуясь только утверждением теоремы 12 и формулами из раздела 6. При рассмотрении алгебры Клиффорда $\mathcal{C}(p, q)$ размерности $n=p+q$ сначала с использованием теоремы 12 узнаем возможные значения параметров $k_{\gamma}$ и $l_{\gamma}$. Затем разбиваем параметр $k_{\gamma}$ всевозможными способами на пары $\left([k p]_{\gamma},[l q]_{\gamma}\right)$ так, что $k_{\gamma}=[k p]_{\gamma}+[k q]_{\gamma}$ и $0 \leqslant[k p]_{\gamma} \leqslant p, 0 \leqslant[k q]_{\gamma} \leqslant q$. Далее по параметрам $\left([k p]_{\gamma},[l q]_{\gamma}\right)$ восстанавливаем дополнительную сигнатуру $\left(r_{\gamma}, s_{\gamma}\right)$ по формулам $r_{\gamma}=[k p]_{\gamma}+q-[k q]_{\gamma}, s_{\gamma}=n-r_{\gamma}$.

Рассмотрим, например, алгебру Клиффорда $\mathcal{C}(1,3)$. Стандартное представление Дирака соответствует случаю $\left(k_{\gamma}, l_{\gamma}\right)=(2,2),\left(r_{\gamma}, s_{\gamma}\right)=(3,1)$. Представление Майорана соответствует случаю $\left(k_{\gamma}, l_{\gamma}\right)=(3,1),\left(r_{\gamma}, s_{\gamma}\right)=(0,4)$. Оставшиеся два случая, указанные в табл. 2 , также реализуются. Случай $\left(k_{\gamma}, l_{\gamma}\right)=(2,2),\left(r_{\gamma}, s_{\gamma}\right)=(1,3)$ получим из представления Дирака, если матрицы, соответствующие генераторам $e^{1}$ и $e^{2}$, умножим на мнимую единицу $i$ и поменяем местами. Случай $\left(k_{\gamma}, l_{\gamma}\right)=(3,1)$, $\left(r_{\gamma}, s_{\gamma}\right)=(2,2)$ получим из представления Майорана, если матрицы, соответствующие генераторам $e^{1}$ и $e^{2}$, умножим на мнимую единицу $i$ и поменяем местами. 
ТАБЛИЦА 2

\begin{tabular}{|c|c|c|c|}
\hline$(p, q)$ & $\left([k p]_{\gamma},[k q]_{\gamma}\right)$ & $\left(k_{\gamma}, l_{\gamma}\right)$ & $\left(r_{\gamma}, s_{\gamma}\right)$ \\
\hline \hline$(1,0)$ & $(1,0)$ & $(1,0)$ & $(1,0)$ \\
\hline$(0,1)$ & $(0,1)$ & $(1,0)$ & $(0,1)$ \\
\hline \hline$(2,0)$ & $(2,0)$ & $(2,0)$ & $(2,0)$ \\
\hline & $(1,0)$ & $(1,1)$ & $(1,1)$ \\
\hline$(1,1)$ & $(1,1)$ & $(2,0)$ & $(1,1)$ \\
\hline & $(1,0)$ & $(1,1)$ & $(2,0)$ \\
\hline & $(0,1)$ & $(1,1)$ & $(0,2)$ \\
\hline$(0,2)$ & $(0,2)$ & $(2,0)$ & $(0,2)$ \\
\hline & $(0,1)$ & $(1,1)$ & $(1,1)$ \\
\hline \hline$(3,0)$ & $(2,0)$ & $(2,1)$ & $(2,1)$ \\
\hline$(2,1)$ & $(1,1)$ & $(2,1)$ & $(1,2)$ \\
\hline & $(2,0)$ & $(2,1)$ & $(3,0)$ \\
\hline$(1,2)$ & $(1,1)$ & $(2,1)$ & $(2,1)$ \\
\hline & $(0,2)$ & $(2,1)$ & $(0,3)$ \\
\hline$(0,3)$ & $(0,2)$ & $(2,1)$ & $(1,2)$ \\
\hline
\end{tabular}

\begin{tabular}{|c|c|c|c|}
\hline$(p, q)$ & $\left([k p]_{\gamma},[k q]_{\gamma}\right)$ & $\left(k_{\gamma}, l_{\gamma}\right)$ & $\left(r_{\gamma}, s_{\gamma}\right)$ \\
\hline \hline$(4,0)$ & $(3,0)$ & $(3,1)$ & $(3,1)$ \\
\hline & $(2,0)$ & $(2,2)$ & $(2,2)$ \\
\hline$(3,1)$ & $(3,0)$ & $(3,1)$ & $(4,0)$ \\
\hline & $(2,1)$ & $(3,1)$ & $(2,2)$ \\
\hline & $(2,0)$ & $(2,2)$ & $(3,1)$ \\
\hline & $(1,1)$ & $(2,2)$ & $(1,3)$ \\
\hline$(2,2)$ & $(2,1)$ & $(3,1)$ & $(3,1)$ \\
\hline & $(1,2)$ & $(3,1)$ & $(1,3)$ \\
\hline & $(2,0)$ & $(2,2)$ & $(4,0)$ \\
\hline & $(1,1)$ & $(2,2)$ & $(2,2)$ \\
\hline & $(0,2)$ & $(2,2)$ & $(0,4)$ \\
\hline$(1,3)$ & $(1,2)$ & $(3,1)$ & $(2,2)$ \\
\hline & $(0,3)$ & $(3,1)$ & $(0,4)$ \\
\hline & $(1,1)$ & $(2,2)$ & $(3,1)$ \\
\hline & $(0,2)$ & $(2,2)$ & $(1,3)$ \\
\hline$(0,4)$ & $(0,3)$ & $(3,1)$ & $(1,3)$ \\
\hline & $(0,2)$ & $(2,2)$ & $(2,2)$ \\
\hline
\end{tabular}

Майорановским сопряжением называется одно из следующих сопряжений (каждый раз, когда существуют соответствующие $\left.C_{ \pm}\right)$:

$$
\begin{array}{lll}
\psi^{M_{+}}=\psi^{\mathrm{T}}\left(C_{+}\right)^{-1}=\left(C_{+}\right)^{-1} \psi^{\sim}, & n \neq 3 & (\bmod 4), \\
\psi^{M_{-}}=\psi^{\mathrm{T}}\left(C_{-}\right)^{-1}=\left(C_{-}\right)^{-1} \psi^{\sim \curlywedge}, & n \neq 1 & (\bmod 4) .
\end{array}
$$

Рассмотрим, к примеру, случай алгебры Клиффорда $\mathcal{C}(1,3)$ и стандартное матричное представление с помощью гамма-матриц Дирака $\gamma^{0}, \gamma^{1}, \gamma^{2}, \gamma^{3}$. Нетрудно проверить, что матрицы $\gamma^{0}$ и $\gamma^{2}$ симметричны, а матрицы $\gamma^{1}$ и $\gamma^{3}$ антисимметричны. Таким образом, имеем $k=l=2$. Получаем $C_{+}=e^{13}, C_{-}=e^{02}, \psi^{M_{+}}=-\psi^{\mathrm{T}} e^{13}$, $\psi^{M_{-}}=\psi^{\mathrm{T}} e^{02}$. В этом случае имеем $C_{ \pm}^{\mathrm{T}}=-C_{ \pm}$, т. е. $\lambda_{ \pm}=-1$.

\section{9. ОБОБЩЕНИЕ ЗАРЯДОВОГО СОПРЯЖЕНИЯ, СПИНОРЫ МАЙОРАНА И МАЙОРАНА-ВЕЙЛЯ В ФОРМАЛИЗМЕ АЛГЕБР КЛИФФОРДА}

Теперь рассмотрим операцию (9) комплексного матричного сопряжения $\overleftarrow{U}$ от элементов алгебры Клиффорда $\mathcal{C}(p, q)$. Данная операция зависит от выбора матричного представления. Не будем путать ее с операцией комплексного сопряжения от элементов алгебры Клиффорда $\overline{e^{a}}=e^{a}$.

Согласно ОТП (см. теорему 7) имеем

$$
\overleftarrow{e^{a}}= \pm B_{ \pm}^{-1} e^{a} B_{ \pm}
$$


причем в случае четного $n$ всегда существуют оба элемента $B_{ \pm}$, а в случае нечетного $n$ - только один из $B_{+}$и $B_{-}$. Можем переписать эти формулы в виде $\overleftarrow{U}=$ $B_{+}^{-1} \bar{U} B_{+}$и $\overleftarrow{U}=B_{-}^{-1} \bar{U}^{\curlywedge} B_{-}$

Сформулируем теоремы об элементах $B_{ \pm}$. Отметим, что формулы $(28)$ и (29) общеизвестны. Мы предлагаем также явный вид элементов $B_{ \pm}$(см. (30) и (31)).

Теорема 13. Элемент $B_{+}$существует в случалх $p-q \neq 3(\bmod 4)$, элемент B_ существует в случаях $p-q \neq 1(\bmod 4)$. Для этих элементов верны формуль

$$
B_{ \pm}^{\mathrm{T}}=\epsilon_{ \pm} B_{ \pm}, \quad \overleftarrow{B_{ \pm}} B_{ \pm}=\epsilon_{ \pm} e
$$

əəe

$$
\epsilon_{+}=\left\{\begin{array}{ll}
+1, & p-q \equiv 0,1,2 \quad(\bmod 8), \\
-1, & p-q \equiv 4,5,6 \quad(\bmod 8),
\end{array} \quad \epsilon_{-}= \begin{cases}+1, & p-q \equiv 0,6,7 \quad(\bmod 8), \\
-1, & p-q \equiv 2,3,4 \quad(\bmod 8)\end{cases}\right.
$$

ТЕОРема 14. В случае матричных представлений, удовлетворяющих условиям (8), (11), имеем явный вид элементов $B_{ \pm}$:

$$
\begin{aligned}
& B_{+}= \begin{cases}\mu_{1} e^{d_{1} \ldots d_{r}}, & r, \text { s нечетны, } \\
\mu_{2} e^{f_{1} \ldots f_{s}}, & r, \text { s четнь }, \\
Z_{1} e^{d_{l} \ldots d_{r}}=Z_{2} e^{f_{1} \ldots f_{s}}, & r \text { нечетно, s четно, }\end{cases} \\
& B_{-}= \begin{cases}\mu_{1} e^{f_{1} \ldots f_{s}}, & r, \text { s нечетны }, \\
\mu_{2} e^{d_{1} \ldots d_{r}}, & r, \text { s четны }, \\
Z_{1} e^{d_{l} \ldots d_{r}}=Z_{2} e^{f_{1} \ldots f_{s}}, & r \text { четно }, \text { м нечетно, }\end{cases}
\end{aligned}
$$

где $\mu_{i}$ - произвольные ненулевые комплексные константы, а $Z_{i}$ - обратимые элементы иентра алгебры Клифборда $\mathcal{C}(p, q)$.

Имеем следующие связи между элементами (в тех случаях, когда каждая тройка элементов существует):

$$
B_{+}=\left(A_{+}^{-1}\right)^{\sim} C_{+}, \quad B_{+}=\left(A_{-}^{-1}\right)^{\sim \curlywedge} C_{-}, \quad B_{-}=\left(A_{-}^{-1}\right)^{\sim} C_{+}, \quad B_{-}=\left(A_{+}^{-1}\right)^{\sim \curlywedge} C_{-} .
$$

ДокАЗАТЕЛЬСтво теОРем 13, 14. Явные формулы для элементов $B_{ \pm}$в случае выделенных матричных представлений следуют из формул (13).

В случае четного $n$ имеем $e^{a}= \pm \overleftarrow{B_{ \pm}^{-1}} \overleftarrow{e} \overleftarrow{B_{ \pm}^{a}}=\overleftarrow{B_{ \pm}^{-1}} B_{ \pm}^{-1} e^{a} B_{ \pm} \overleftarrow{B_{ \pm}}$, т. е. $B_{ \pm} \overleftarrow{B_{ \pm}}=\epsilon_{ \pm} e$ Значит, $\epsilon_{ \pm} \in \mathbb{R}$. Поскольку $\operatorname{Det}\left(B_{ \pm}\right)=1$, то $\epsilon_{ \pm}=1,-1$. Кроме того, $\left(B_{ \pm}\right)^{\dagger} B_{ \pm}=e$ и $B_{ \pm}^{\mathrm{T}}=\left(\overleftarrow{B_{ \pm}}\right)^{\dagger}=\left(\epsilon_{ \pm} B_{ \pm}^{-1}\right)^{\dagger}=\epsilon_{ \pm} B_{ \pm}$.

В случае нечетного $n$ можно получить те же формулы (28), если воспользоваться тем, что в случае выделенных матричных представлений элементы $B_{ \pm}$всегда найдутся среди элементов базиса $e^{A}$ (см. формулы (30) и (31)).

Элементы $B_{ \pm}$могут быть выражены через элементы $A_{ \pm}$и $C_{ \pm} . \mathrm{K}$ примеру, всегда, кроме случая четного $p$ и нечетного $q, n \equiv 3(\bmod 4)$, из $U^{\dagger}=A_{+}^{-1} U^{\ddagger} A_{+}$ и $U^{\mathrm{T}}=C_{+}^{-1} U^{\sim} C_{+}$получаем $\overleftarrow{U}=C_{+}^{-1}\left(A_{+}^{-1} U^{\ddagger} A_{+}\right)^{\sim} C_{+}=C_{+}^{-1} A_{+}^{\sim} \bar{U}\left(A_{+}^{-1}\right)^{\sim} C_{+}$, а значит, $B_{+}=\left(A_{+}^{-1}\right) \sim C_{+}$. Аналогично доказываются три другие формулы из утверждения теоремы. 
Теперь получим явный вид для коэффициентов $\epsilon_{ \pm}$. Проведем выкладку в случае, когда существуют $A_{+}$и $C_{+}$:

$$
\begin{aligned}
\epsilon_{+} & =B_{+}^{\mathrm{T}} B_{+}^{-1}=\left(\left(A_{+}^{-1}\right)^{\sim} C_{+}\right)^{\mathrm{T}}\left(\left(A_{+}^{-1}\right)^{\sim} C_{+}\right)^{-1}=C_{+}^{\mathrm{T}}\left(A_{+}^{-1}\right)^{\sim \mathrm{T}} C_{+}^{-1} A_{+}^{\mathrm{T}}= \\
& =\lambda_{+} C_{+} C_{+}^{-1} A_{+}^{-1} C_{+} C_{+}^{-1} A_{+}^{\sim}=\lambda_{+} A_{+}^{-1} A_{+}^{\sim} .
\end{aligned}
$$

В случае, когда существуют $A_{-}$и $C_{-}$, проводим аналогичную выкладку и получаем $\epsilon_{+}=\lambda_{-} A_{-}^{-1} A_{-}^{\sim \curlywedge}$. Когда существуют $A_{-}$и $C_{+}$, получаем $\epsilon_{-}=\lambda_{+} A_{-}^{-1} A_{-}^{\sim}$. Когда существуют $A_{+}$и $C_{-}$, получаем $\epsilon_{-}=\lambda_{-} A_{+}^{-1} A_{+}^{\sim \curlywedge}$. Зная значения $\lambda_{ \pm}$(зависящие от $n$ ) и значения $A_{ \pm}$(зависящие от четности $p$ и $q$ ), получаем из этих четырех формул (которые охватывают все возможные случаи) значения констант $\epsilon_{ \pm}$в зависимости от $p$ и $q(29)$.

Заметим, что несмотря на то что элементы $B_{ \pm}$зависят от матричного представления, их симметричность (28) определяется только сигнатурой пространства $p-q(29)$.

Рассмотрим две операции зарядового сопряжения

$$
\begin{array}{lll}
\psi^{\mathrm{ch}_{+}}=B_{+} \overleftarrow{\psi}=\bar{\psi} B_{+}, & p-q \neq 3 & (\bmod 4) \\
\psi^{\mathrm{ch}_{-}}=B_{-} \overleftarrow{\psi}=\bar{\psi}^{\curlywedge} B_{-}, & p-q \neq 1 & (\bmod 4)
\end{array}
$$

Теорема 15. Имеем следующие эквивалентные определения зарядового сопряжения (когда существуют соответствующие элементы $C_{ \pm}$и $\left.A_{ \pm}\right)$:

$$
\begin{array}{ll}
\psi^{\mathrm{ch}_{+}}=C_{+}\left(\psi^{\mathrm{D}_{+}}\right)^{\mathrm{T}}, & \psi^{\mathrm{ch}_{+}}=C_{-}\left(\psi^{\mathrm{D}_{-}}\right)^{\mathrm{T}}, \\
\psi^{\mathrm{ch}_{-}}=C_{+}\left(\psi^{\mathrm{D}_{-}}\right)^{\mathrm{T}}, & \psi^{\mathrm{ch}_{-}}=C_{-}\left(\psi^{\mathrm{D}_{+}}\right)^{\mathrm{T}} .
\end{array}
$$

Доказательство. Учитывая связь между элементами $A_{ \pm}, B_{ \pm}$и $C_{ \pm}(32)$, получаем

$$
\psi^{\mathrm{ch}_{+}}=B_{+} \overleftarrow{\psi}=\left(A_{+}^{-1}\right)^{\sim} C_{+} \overleftarrow{\psi}=C_{+}\left(A_{+}^{-1}\right)^{\mathrm{T}} C_{+}^{-1} C_{+} \overleftarrow{\psi}=C_{+}\left(\psi^{\dagger} A_{+}^{-1}\right)^{\mathrm{T}}=C_{+}\left(\psi^{\mathrm{D}_{+}}\right)^{\mathrm{T}}
$$

Аналогично можно получить остальные три формулы.

Рассмотрим в качестве примера алгебру Клиффорда $\mathcal{C}(1,3)$ и стандартное матричное представление с помощью гамма-матриц Дирака. Поскольку матрицы $\gamma^{0}$, $\gamma^{1}$ и $\gamma^{3}$ вещественны, а матрица $\gamma^{2}$ чисто мнимая, то $r=3, s=1, \epsilon_{+}=-1, \epsilon_{-}=1$ $($ так как $p-q=6(\bmod 8))$ и $B_{+}=e^{013}, B_{-}=e^{2}, \psi^{\mathrm{ch}_{+}}=e^{013 \overleftarrow{\psi}}, \psi^{\mathrm{ch}_{-}}=e^{2} \overleftarrow{\psi}$.

Майорановские спиноры и псевдомайорановские спиноры (часто называют просто спинорами Майорана) определяются следующим образом:

$$
E_{\mathrm{M}}=\left\{\psi \in E_{\text {Dirac }} \mid \psi^{\mathrm{ch}_{-}}= \pm \psi\right\}, \quad E_{\mathrm{psM}}=\left\{\psi \in E_{\text {Dirac }} \mid \psi^{\mathrm{ch}_{+}}= \pm \psi\right\}
$$

В завершение приведем общеизвестные утверждения о реализации спиноров Майорана и Майорана-Вейля в случае произвольных размерностей и сигнатур пространства.

ПРеДЛОЖЕнИЕ 1. Спинорь Майорана реализуются в случае $p-q \equiv 0,6$, $7(\bmod 8)$, а псевдомайорановские спиноры - в случае $p-q \equiv 0,1,2(\bmod 8)$. 
ДокАЗАТЕльство. Для псевдомайорановских спиноров получаем

$$
\psi= \pm B_{+} \overleftarrow{\psi}, \quad \pm B_{+}^{-1} \psi=\overleftarrow{\psi}= \pm \overleftarrow{B}_{+} \psi= \pm \epsilon_{+} B_{+}^{-1} \psi, \quad\left(1-\epsilon_{+}\right) \psi=0, \quad \epsilon_{+}=1
$$

Аналогично для спиноров Майорана получаем $\epsilon_{-}=1$. Далее пользуемся формулами (29).

Заметим, что в случае $p-q \equiv 0,1,2(\bmod 8)$ алгебра Клиффорда $\mathcal{C} \mathbb{R}^{\mathbb{R}}(p, q)$ изоморфна алгебре вещественных матриц, а в случае $p-q \equiv 0,6,7(\bmod 8)$ то же самое можно сказать про алгебру Клиффорда $\mathcal{C} \ell^{\mathbb{R}}(q, p)$ (см. теорему 2).

Левые и правые спиноры Майорана-Вейля определяются следующим образом:

$$
\begin{aligned}
E_{\mathrm{M}-\mathrm{W}}^{\mathrm{L}} & =\left\{\psi \in E_{\text {Weyl }}^{\mathrm{L}} \mid \psi^{\mathrm{ch}_{+}}= \pm \psi\right\}, \quad E_{\mathrm{M}-\mathrm{W}}^{\mathrm{R}}=\left\{\psi \in E_{\text {Weyl }}^{\mathrm{R}} \mid \psi^{\mathrm{ch}_{-}}= \pm \psi\right\}, \\
E_{\mathrm{psM}-\mathrm{W}}^{\mathrm{L}} & =\left\{\psi \in E_{\text {Weyl }}^{\mathrm{L}} \mid \psi^{\mathrm{ch}_{-}}= \pm \psi\right\}, \quad E_{\mathrm{psM}-\mathrm{W}}^{\mathrm{R}}=\left\{\psi \in E_{\text {Weyl }}^{\mathrm{R}} \mid \psi^{\mathrm{ch}_{+}}= \pm \psi\right\} .
\end{aligned}
$$

ПРЕДЛОЖЕНИЕ 2. Спиноры Майорана-Вейля реализуются в случае $p-q=0$ $(\bmod 8)$.

ДокАЗАтЕЛьство. Спиноры Вейля реализуются только в случае четного $n$, причем $\omega=e^{1 \ldots n}$ для $p-q=0(\bmod 4)$ и $\omega=i e^{1 \ldots n}$ для $p-q=2(\bmod 4)($ см. раздел 5$)$. Покажем, что при $p-q=2(\bmod 4)$ спиноры Майорана-Вейля не реализуются. Тогда согласно предложению 1 получим только случаи $p-q=0(\bmod 8)$. Действительно, пусть $\omega=i e^{1 \ldots n}$. Тогда условие левых спиноров Вейля $i e^{1 \ldots n} \psi=-\psi$ при действии операции комплексного матричного сопряжения переходит в условие правых спиноров Вейля $i e^{1 \ldots n} \psi=\psi$ (действительно, имеем $B_{+}^{-1}\left(-i e^{1 \ldots n}\right) B_{+} \overleftarrow{\psi}=-\overleftarrow{\psi}$ и $B_{+} \overleftarrow{\psi}= \pm \psi$ ), и мы приходим к противоречию. Аналогичную выкладку можно провести с элементом $B_{-}$, используя $B_{-} \overleftarrow{\psi}= \pm \psi$

Отметим связь результатов, полученных в настоящей работе, с другими результатами. В литературе принято уделять наибольшее внимание случаю сигнатур $(1, n-1)$, тогда как в настоящей работе изложен формализм спиноров для произвольных сигнатур $(p, q)$. В работах [5], [7], [9] изучаются (только в случае сигнатур $(1, n-1))$ свойства трех элементов, каждый из которых соответствует одному из элементов каждого вида $A_{ \pm}, B_{ \pm}, C_{ \pm}$настоящей статьи. В работе [8] был впервые рассмотрен случай произвольной сигнатуры $(p, q)$ и по одному из элементов каждого вида $\left(A_{ \pm}, B_{ \pm}, C_{ \pm}\right)$. В статьях [6], [18] рассматриваются по два элемента каждого вида и доказываются основные свойства этих элементов. Явный вид элементов $A_{ \pm}$, $B_{ \pm}, C_{ \pm}$, зависящий от матричного представления, дан в настоящей статье (см. теоремы $9,11,14)$.

Отметим также, что мы обсуждаем математические структуры и конструкции. Соотнесение предлагаемых в статье математических конструкций с объектами реального мира (элементарными частицами) выходит за рамки настоящего исследования. Целью настоящей работы является математическое развитие теории.

Некоторые результаты, касающиеся существования спиноров Вейля, Майорана и Майорана-Вейля для тех или иных размерностей и сигнатур пространства, применяются в теории суперсимметрии. В случае сигнатур $p-q=0(\bmod 8)$ в качестве суперзарядов часто берут спиноры Майорана-Вейля. В случае сигнатур 
$p-q=0,1,2,6,7(\bmod 8)$ рассматривают спиноры Майорана и псевдомайорановские спиноры. В связи с рассмотрением спиноров разного типа суперсимметричное расширение алгебры Пуанкаре строится по-разному в зависимости от размерности и сигнатуры пространства (см. приведенные в работах [5]-[19] ссылки).

Благодарности. Автор выражает глубокую благодарность И.В. Воловичу, Н.Г. Марчуку и рецензенту за полезные замечания. Работа выполнялась в рамках Программы поддержки ведущих научных школ (грант НШ-2928.2012.1) и при поддержке Министерства образования и науки РФ (соглашение 8215).

\section{Список литературы}

[1] W. Pauli, Ann. Inst. H. Poincaré, 6:2 (1936), 109-136.

[2] Н. Н. Боголюбов, Д. В. Ширков, Квантовые поля, Наука, М., 1980.

[3] Н. Н. Боголюбов, А.А. Логунов, А. И. Оксак, И. Т. Тодоров, Общие принципъ квантовой теории поля, Наука, М., 1987.

[4] M. A. Naimark, A. I. Stern, Theory of Group Representations, Grundlehren der Mathematischen Wissenschaften [Fundamental Principles of Mathematical Sciences], 246, Springer, Berlin, 1982.

[5] P. C. West, "Supergravity, brane dynamics and string duality", Duality and Supersymmetric Theories (Cambridge, England, April 7-18, 1997), eds. D. I. Olive, P. C. West, Cambridge Univ. Press, Cambridge, 1999, 147-266, arXiv: hep-th/9811101.

[6] Y. Tanii, Introduction to supergravities in diverse dimensions, arXiv: hep-th/9802138.

[7] Дж. Шерк, "Расширенная суперсимметрия и теория расширенной супергравитации", Геометрические идеи в физике, Сб. статей, ред. Ю. И. Манин, Мир, М., 1983, 203-239.

[8] T. Kugo, P. Townsend, Nucl. Phys. B, 221:2 (1983), 357-380.

[9] F. Gliozzi, J. Sherk, D. Olive, Nucl. Phys. B, 122:2 (1977), 253-290.

[10] B. DeWitt, Supermanifolds, Cambridge Univ. Press, Cambridge, 1984.

[11] И. Я. Арефьева, И. В. Волович, УФН, 146:4 (1985), 655-681.

[12] В. С. Владимиров, И. В. Волович, ТМФ, 59:1 (1984), 3-27.

[13] В. С. Владимиров, И. В. Волович, ТМФ, 60:2 (1984), 169-198.

[14] J. Strathdee, Internat. J. Modern Phys. A, 2:1 (1987), 273-300.

[15] P. G. O. Freund, Introduction to Supersymmetry, Springer, Berlin, 1986.

[16] П. Уэст, Введение в суперсимметрию и супергравитацию, Мир, М., 1989.

[17] А. В. Галажинский, Введение в суперсимметрию, Изд. Томск. политехн. ун-та, Томск, 2008.

[18] M. Rausch de Traubenberg, "Clifford Algebras in Physics", Lectures of the 7th International Conference on Clifford Algebras and their Applications - ICCA-7 (Toulouse, France, May 19-29, 2005), Institut de Mathématiques de Toulouse, Toulouse, 2005, arXiv: hep-th/0506011.

[19] F. Quevedo, Cambridge lectures on supersymmetry and extra dimensions, arXiv: 1011.1491.

[20] Д. С. Широков, Докл. РАН, 440:5 (2011), 607-610.

[21] N. G. Marchuk, D.S. Shirokov, Local generalized Pauli's theorem, arXiv: 1201.4985.

[22] W. K. Clifford, Amer. J. Math., 1:4 (1878), 350-358.

[23] H. Grassmann, Die Lineale Ausdehnungslehre, ein neuer Zweig der Mathematik, Verlag von Otto Wigand, Leipzig, 1844.

[24] W. R. Hamilton, Philos. Mag., 25 (1844), 489-495.

[25] C. Chevalley, Collected Works, v. 2: The Algebraic Theory of Spinors and Clifford Algebras, Springer, Berlin, 1997. 
[26] M. Riesz, "Sur certaines notions fondamentales en théorie quantique relativiste", $C . R$. Dixième Congrès Math. Scandinaves (Gjellerups Forlag, Copenhagen, July 1946), Jul. Gjellerups Forlag, Copenhagen, 1947, 123-148; "L'équation de Dirac en relativité générale", Collected Papers, eds. L. Gårding, L. Hörmander, Springer, Berlin, 1988, 814-832.

[27] P. A. M. Dirac, Proc. Roy. Soc. London A, 117:778 (1928), 610-624.

[28] D. Hestenes, G. Sobczyk, Clifford Algebra to Geometric Calculus. A Unified Language for Mathematical Physics, Reidel, Dordrecht, 1984.

[29] Н. Г. Марчук, Уравнения теории поля и алгебры Клиффорда, РХД, Ижевск, 2009.

[30] P. Lounesto, Clifford Algebras and Spinors, London Mathematical Society Lecture Note Series, 286, Cambridge Univ. Press, Cambridge, 2001.

[31] G. Juvet, Comment. Math. Helv., 2:1 (1930), 225-235.

[32] F. Sauter, Z. Phys., 63:11-12 (1930), 803-814; 64:5-6 (1930), 295-303.

[33] I. M. Benn, R. W. Tucker, An Introduction to Spinors and Geometry with Applications in Physics, Adam Hilger, Bristol, 1987.

[34] П. К. Рашевский, УМН, 10:2(64) (1955), 3-110.

[35] Ю. Б. Румер, Спинорный анализ, ОНТИ, М.-Л., 1936.

[36] N. G. Marchuk, D. S. Shirokov, Adv. Appl. Clifford Algebr., 18:2 (2008), 237-254.

[37] D. S. Shirokov, Concepts of trace, determinant and inverse of Clifford algebra elements, arXiv: 1108.5447.

Поступила в редакцию 18.06.2012,

после доработки 2.11.2012 